\title{
Review:
}

\section{Mathematically modeling fixed-bed adsorption in aqueous systems"}

\author{
Zhe XU, Jian-guo CAI, Bing-cai PAN ${ }^{\dagger \neq}$ \\ (State Key Laboratory of Pollution Control and Resource Reuse, School of the Environment, Nanjing University, Nanjing 210023, China) \\ †E-mail: bcpan@nju.edu.cn
}

Received Jan. 18, 2013; Revision accepted Jan. 30, 2013; Crosschecked Feb. 22, 2013

\begin{abstract}
Adsorption is one of the widely used processes in the chemical industry environmental application. As compared to mathematical models proposed to describe batch adsorption in terms of isotherm and kinetic behavior, insufficient models are available to describe and predict fixed-bed or column adsorption, though the latter one is the main option in practical application. The present review first provides a brief summary on basic concepts and mathematic models to describe the mass transfer and isotherm behavior of batch adsorption, which dominate the column adsorption behavior in nature. Afterwards, the widely used models developed to predict the breakthrough curve, i.e., the general rate models, linear driving force (LDF) model, wave propagation theory model, constant pattern model, Clark model, Thomas model, Bohart-Adams model, Yoon-Nelson model, Wang model, Wolborska model, and modified dose-response model, are briefly introduced from the mechanism and mathematical viewpoint. Their basic characteristics, including the advantages and inherit shortcomings, are also discussed. This review could help those interested in column adsorption to reasonably choose or develop an accurate and convenient model for their study and practical application.
\end{abstract}

Key words: Column adsorption, Modeling, Fixed-bed adsorption, Breakthrough curve

doi:10.1631/jzus.A1300029 Document code: A CLC number: X1

\section{Introduction}

Adsorption is a widely used method to treat industrial waste gas and effluent due to its low cost, high efficiency and easy operation. Particularly, the adsorption process is suitable for decontaminating those compounds of low concentration or high toxicity, which are not readily treated by biological processes. Based on the operation mode, adsorption can be generally classified into static adsorption and dynamic adsorption. Static adsorption, also called batch adsorption, occurs in a closed system containing a

\footnotetext{
ॠ Corresponding author

* Project supported by the National Natural Science Foundation of China (No. 21177059), the Science Foundation of Ministry of Education of China (No. 20120091130005), the Natural Science Foundation of Jiangsu Province of China (No. BK2012017), the Program for New Century Excellent Talents in University of China (No. NCET10-0490), and the Changjiang Scholars Innovative Research Team in University (No. IRT1019), China

(C) Zhejiang University and Springer-Verlag Berlin Heidelberg 2013
}

desired amount of adsorbent contacting with a certain volume of adsorbate solution, while dynamic adsorption usually occurs in an open system where adsorbate solution continuously passes through a column packed with adsorbent. For column adsorption, how to determine the breakthrough curve is a very important issue because it provides the basic but predominant information for the design of a column adsorption system. Without the information of the breakthrough curve one cannot determine a rational scale of a column adsorption for practical application. There are two widely used approaches to obtain the breakthrough curve of a given adsorption system: direct experimentation or mathematical modeling. The experimental method could provide a direct and concise breakthrough curve of a given system. However, it is usually a time-consuming and economical undesirable process, particularly for the trace contaminants and long residence time. Also, it greatly depends upon the experimental conditions, such as ambient temperature and residence time. 
Comparatively, mathematical modeling is simple and readily realized with no experimental apparatus required, and thus, it has attracted increasing interest in the past decades.

Currently, a variety of mathematical models have been used to describe and predict the breakthrough curves of a column adsorption system in liquid or gaseous phase (Abu-Lail et al., 2012; Cheknane et al., 2012; Meng et al., 2012; Nwabanne and Igbokwe, 2012; Yi et al., 2012; Zhao et al., 2012), but there is still lack of a comprehensive review of these models. The main objective of the present review is to introduce the modeling of dynamic adsorption in liquid phase. Different from the gas-solid adsorption, liquid-solid adsorption is more theoretically difficult to give an unambiguous description because the solvent accompanies more intricate interaction between the species involved. Moreover, the salvation effect results in a more complicated behavior of the process. To model a liquid-solid column adsorption, it is necessary to divide it into four basic steps (Fig. 1): (1) liquid phase mass transfer including convective mass transfer and molecular diffusion; (2) interface diffusion between liquid phase and the exterior surface of the adsorbent (i.e., film diffusion); (3) intrapellet mass transfer involving pore diffusion and surface diffusion; and, (4) the adsorption- desorption reaction (Crittenden and Weber, 1978; Crittenden et al., 1986; Helfferich, 1995).

(1) Liquid phase mass transfer. Molecules or ions in the column can move in both axial and radial directions. For simplification, it is common to postulate that all cross-sections are homogeneous and the radial movement could be neglected. Thus, a macroscopic mass conservation equation is acquired to represent the relationship between the corresponding variations (i.e., concentration of the adsorbed adsorbate $q$; concentration of the bulk solution $C$; distance to the inlet $z$; superficial velocity $u$; and axial dispersion coefficient $D_{\mathrm{z}}$ (if the axial dispersion is not ignored)). Regarding a control volume as shown in Fig. 2, one has (Costa and Rodrigues, 1985; Tien, 1994; Fournel et al., 2010)

$$
\varepsilon \frac{\partial C}{\partial t}+u \frac{\partial C}{\partial z}+(1-\varepsilon) \rho_{\mathrm{a}} \frac{\partial q}{\partial t}=D_{z} \frac{\partial^{2} C}{\partial z^{2}}
$$

where initial and boundary conditions are

$$
\begin{aligned}
& t=0 \rightarrow C(z, t)=0, \\
& t=0 \rightarrow q(z, t)=0, \\
& z=0 \rightarrow C(0, t=0)=0, C(0, t>0)=C_{\mathrm{F}}, \\
& z=H \rightarrow \frac{\partial C}{\partial z}=0 .
\end{aligned}
$$

When the axial dispersion is ignored,

$$
\varepsilon \frac{\partial C}{\partial t}+u \frac{\partial C}{\partial z}+(1-\varepsilon) \rho_{\mathrm{a}} \frac{\partial q}{\partial t}=0
$$

The initial and boundary conditions turn to

$$
\begin{aligned}
& t=0 \rightarrow C(z, t)=0, \\
& z=0 \rightarrow C=C_{\mathrm{F}}+\frac{D_{z} \varepsilon}{u} \frac{\partial C}{\partial z}, \\
& z=H \rightarrow \frac{\partial C}{\partial z}=0,
\end{aligned}
$$

where $\varepsilon$ is the bed porosity, $t$ is the time, $\rho_{\mathrm{a}}$ is the adsorbent density, $C_{\mathrm{F}}$ is the initial concentration of the influent, and $H$ is the bed height.

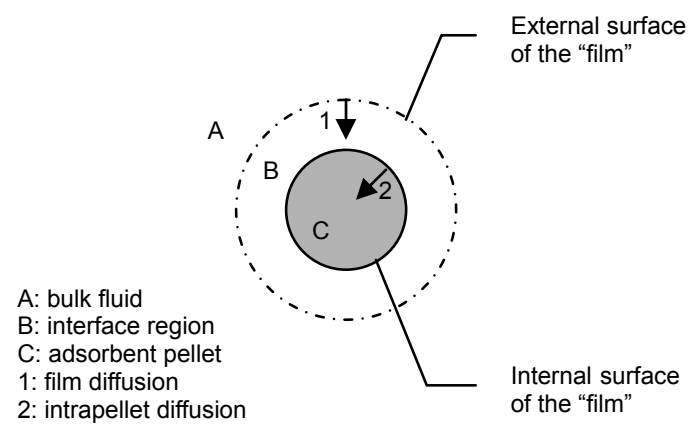

Fig. 1 Macroscopic adsorption process of an adsorbent pellet

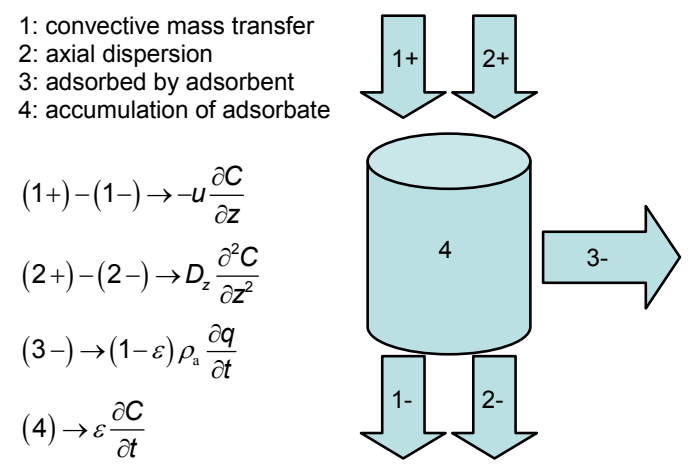

Fig. 2 Schematic diagram of the mass conservation of a control volume 
Eqs. (1) and (2) are based on the following assumptions: (1) the process is isothermal; (2) no chemical reaction occurs in the column; (3) the packing material is made of porous particles that are spherical and uniform in size; (4) the bed is homogenous and the concentration gradient in the radial direction of the bed is negligible; (5) the flow rate is constant and invariant with the column position (Warchoł and Petrus, 2006); and, (6) the activity coefficient of each species is unity.

(2) Film diffusion. The driving force of film diffusion is the concentration gradient located at the interface region between the exterior surface of adsorbent pellets and the bulk solution. As the first step of adsorption, film diffusion predominates the overall uptake rate to some extent and even becomes the rate control step in some cases. The flux film diffusion can be expressed in linear form by multiplying its driving force and the phenomenological coefficient (Tien 1994; Fournel et al., 2010):

$$
\frac{\mathrm{d} q}{\mathrm{~d} t}=J_{\mathrm{f}}=k_{\mathrm{f}} a\left(C-C_{\mathrm{s}}\right)
$$

where $J_{\mathrm{f}}$ is the mass transfer flux, $a$ is the volumetric surface area, $C_{\mathrm{s}}$ is the adsorbate concentration at the exterior surface of adsorbent, and $k_{\mathrm{f}}$ is the film diffusion coefficient. It is generally known that increasing the flow rate will decrease the film thickness and resistance, whereas larger film resistance can be caused by packing with smaller adsorbent pellets due to the extension of the exterior surface area.

(3) Intrapellet diffusion and reaction. As shown in Fig. 3, surface diffusion and pore diffusion proceed in parallel accompanying with Knudsen diffusion and the adsorption reactions. Of note, when the pore size is only slightly larger than the diameter of adsorbate ions or molecules, the Knudsen diffusion begins to play a significant role as shown in Fig. 3b.

Generally speaking, the film diffusion driven solely by the concentration gradient can be expressed in a routine form (Eq. (3)), and the intrapellet diffusion, which is more complex and diverse, is the keystone of modeling dynamic adsorption. Pore diffusion, surface diffusion and reaction are involved in intrapellet transfer simultaneously, and a set of equations could be set to consider all the possible mechanisms. Moreover, consideration of the heterogeneity and Knudsen diffusion will tend to cause dramatic complexity and make the process very tedious. Hence, it is urgent to simplify such a process by making appropriate assumptions based on specific characteristics of the system, and several models were proposed based on different simplifications indeed.

Most mathematical models to predict a breakthrough curve have (or are acquired by) the same composition, i.e., (a) macroscopic mass conservation equation; (b) adsorption kinetic equation (sometimes including a set of equations); and (c) equilibrium relationship. Among these composition, (a) and (b) have been briefly discussed above. Logically, we introduce the equilibrium relationship (isotherm) in the following sections.
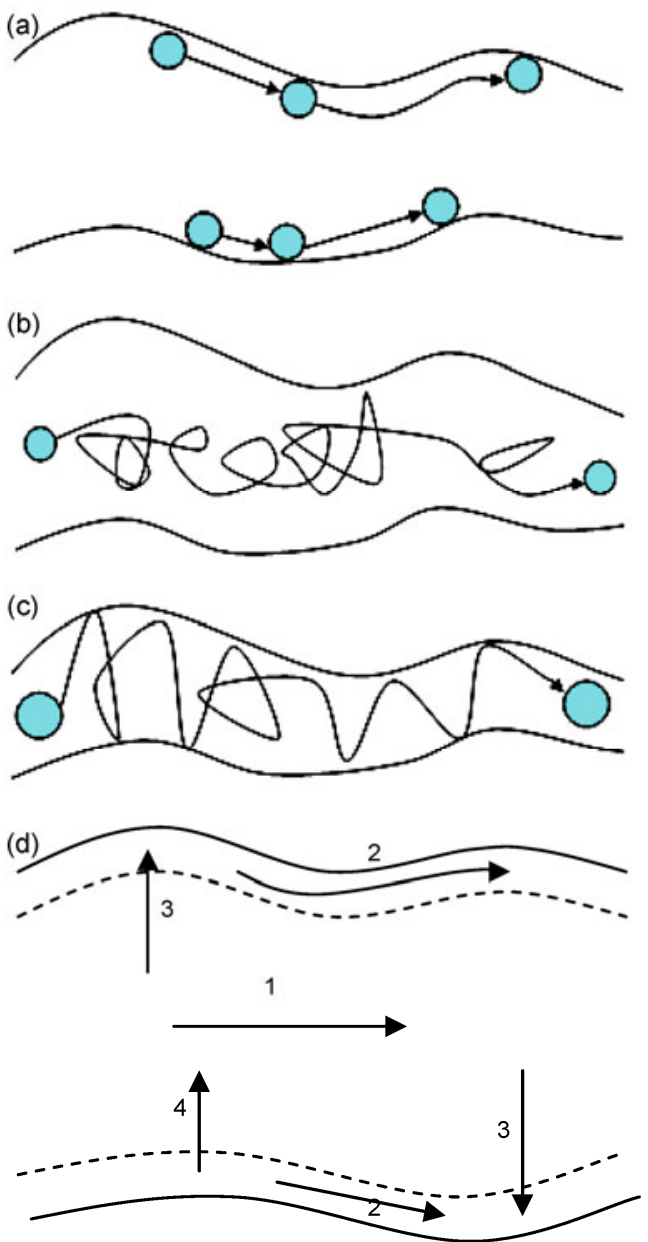

Fig. 3 Macroscopic schematic illustration of basic diffusion and adsorption steps inside the pore

(a) Surface diffusion; (b) Pore diffusion; (c) Pore diffusion with significant Knudsen diffusion; (d) Combination of intrapellet diffusion and adsorption. 1: pore diffusion; 2: surface diffusion; 3: adsorption; 4: desorption 


\section{Single-component isotherms}

As we have illustrated, the general way to predict the breakthrough curve is to solve a set of partial differential equations which consist of a macroscopic mass conservation equation, uptake rate equation (sometimes including a set of equations), and isotherm equation. Obviously, as a prerequisite of modeling of the dynamic adsorption, the choice of the isotherm style will directly affect the effect of mathematic modeling. Although several methods have been adopted to determine the isotherm, the most widely used one is the conventional static method proceeding in a closed system. Actually, due to the complexity of the structure of adsorbent and the interaction between each corpuscle, isotherms can present diverse shapes. As shown in Fig. 4, Giles et al. (1960) classified different isotherms into four types (S, L, H and C types). Malek and Farooq (1996) suggested that there are three fundamental means to formulate an isotherm: dynamic equilibrium between adsorption and desorption, thermodynamic equilibrium between phases and species, and adsorption potential theory. Although researchers have developed various isotherm models in the past decades, it is clear that none of them fit well with all cases, and thus, one has to determine the best suitable isotherm experimentally. Morgenstern (2004) reviewed the means to derive the isotherm by both batch method and adsorption-desorption method. Here we do not intend to give a detailed discussion of various isotherms but only concisely introduce several widespread models.

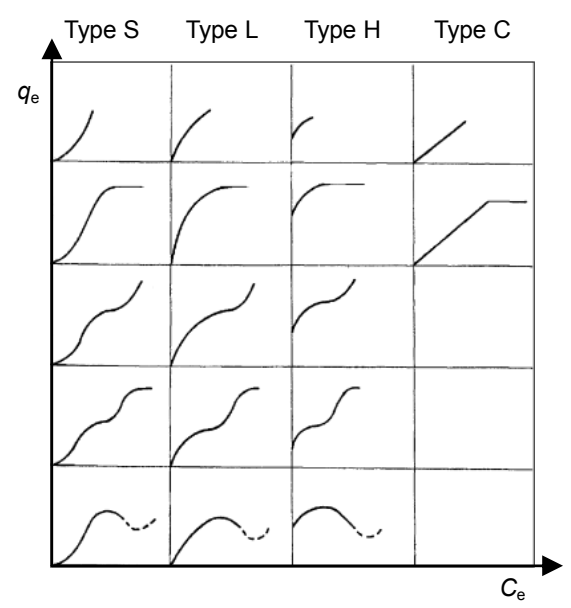

Fig. 4 Adsorption isotherms classified by Giles et al. (1960)

\subsection{Langmuir isotherm}

The Langmuir isotherm assumes: (1) the adsorption process takes place as monolayer adsorption (chemical adsorption); (2) the surface of adsorbent pellets or each adsorption site is homogeneous; and, (3) the adsorption heat does not vary with the coverage. In other words, in terms of the Langmuir isotherm, adsorption takes place when a free adsorbate molecule collides with an unoccupied adsorption site and each adsorbed molecule has the same percentage to desorption (Langmuir, 1916). The model can be written as

$$
q_{\mathrm{e}}=\frac{q_{\mathrm{m}} b C_{\mathrm{e}}}{1+b C_{\mathrm{e}}}
$$

where $q_{\mathrm{e}}$ is the value of $q$ at equilibrium, $q_{\mathrm{m}}$ is the maximum adsorptive capacity, $C_{\mathrm{e}}$ is the concentration of adsorbate in liquid phase at equilibrium, and $b$ is the Langmuir constant. Certainly, we can obtain a linear form of the Langmuir model

$$
\frac{C_{\mathrm{e}}}{q_{\mathrm{e}}}=\frac{1}{q_{\mathrm{m}} b}+\frac{C_{\mathrm{e}}}{q_{\mathrm{m}}}
$$

where

$$
b=k_{\mathrm{a}} / k_{\mathrm{d}},
$$

where $k_{\mathrm{a}}$ refers to the adsorption rate coefficient of the Langmuir kinetic model, and $k_{\mathrm{d}}$ is the desorption rate coefficient (Azizian, 2004). Despite the reversible adsorption nature of the Langmuir model, it sometimes fits irreversible adsorption well. Because of its simple form and well fitting performance, the Langmuir isotherm has become one of the most popular models in adsorption studies.

\subsection{Freundlich isotherm}

Another most widely used model is the Freundlich isotherm. Comparing with the Langmuir isotherm, the Freundlich isotherm does not have much limitation, i.e., it can deal with both homogeneous and heterogeneous surfaces, and both physical and chemical adsorption. Especially, this model frequently succeeds in depicting the adsorption behavior of organic compounds and reactive matters. The Freundlich isotherm is expressed as 


$$
q_{\mathrm{e}}=K C_{\mathrm{e}}^{1 / n},
$$

and its linear form is

$$
\ln q_{\mathrm{e}}=\ln K+\frac{1}{n} \ln C_{\mathrm{e}},
$$

where $K$ and $n$ are the parameters to be determined. Though the Freundlich isotherm is one of the earliest empirical correlation, it could be deduced from the assumption that $Q_{\mathrm{a}}=Q_{\mathrm{a}, 0}-a_{\mathrm{f}} \ln \theta$, where $Q_{\mathrm{a}}$ is the differential heat of adsorption, $\theta$ is the coverage, $Q_{\mathrm{a}, 0}$ is the value of $Q_{\mathrm{a}}$ at $\theta=0$, and $a_{\mathrm{f}}$ is a constant. According to (Haghseresht and $\mathrm{Lu}, 1998$ ), the surface heterogeneity and type of adsorption can be roughly estimated by the Freundlich parameters.

\subsection{Other isotherms}

Besides the above two isotherms, adsorption equilibrium can also be described by other isotherms such as the Sips model (Sips, 1948), Toth model (Toth, 1971), and the Brunauer-Emmett-Teller (BET) model (Brunauer et al., 1938). One must note that the lower prevalence of these isotherms do not mean less functionality, e.g., the Dubinin-Radushkevich isotherm is able to calculate the mean adsorption free energy from which the prediction of adsorption type is available (Dubinin and Radushkevich, 1947); the Temkin isotherm allows one to estimate the effect of temperature (Temkin and Pyzhev, 1940). The single-component isotherms have been summarized in several studies (Foo and Hameed, 2010). Depending on the linear expression of each isotherm, all the isotherm parameters could be acquired by linear regression, and several commonly used error functions are outlined in Table 1.

\section{Multi-component isotherms}

When a variety of pollutants is present with the target pollutant in solution, the equilibrium relationship of any component may not fit the single-component isotherms since competitive adsorption occurs between different species. In order to solve the problem, multi-component isotherms were developed, among which the ideal adsorbed solution theory (IAST) model based on the equivalence of the spreading pressure, $\pi$, of each component is one of the most reliable iso- therms (Myers and Prausnitz, 1965; Radke and Prausnitz, 1972; Hand et al., 1985). Namely,

$$
\pi_{i}=\pi_{j} .
$$

The spreading pressure is a surface chemistry terminology referring to the difference of surface tension of the solvent-solid interface and solutionssolid interface. According to the Gibbs adsorption formula

$$
\Gamma_{i}=\frac{1}{R T} \frac{\mathrm{d} \pi_{i}}{\ln a_{i}}
$$

where $\Gamma_{i}$ is the adsorptive capacity per surface area of species $i, R$ is the ideal gas constant, $T$ is the temperature, and $a_{i}$ is the activity of solute $i$. Assuming that the activity coefficient of each solute is unity, and $a_{i}$ could be substituted by $C_{i}$ which refers to the concentration of solute $i$. Thus, we can rewrite Eq. (10) as (Myers and Prausnitz, 1965)

$$
\pi_{i}\left(C_{i}\right)=\frac{R T}{a} \int_{0}^{C_{i}^{*}} \frac{q_{i}}{C_{i}} \mathrm{~d} C_{i},
$$

or

$$
\pi_{i}\left(q_{i}\right)=\frac{R T}{a} \int_{0}^{-q_{i}^{*}} \frac{\mathrm{d} \log C_{i}}{\mathrm{~d} \log q_{i}} \mathrm{~d} q_{i},
$$

where $q_{i}{ }^{*}$ and $C_{i}{ }^{*}$ are respectively the solid phase and liquid phase concentrations of species $i$ to generate the surface tension that is equal to $\pi_{i}$. Eqs. (13)-(18)

Table 1 Lists of some widely used error functions

\begin{tabular}{lc}
\hline \multicolumn{1}{c}{ Method } & Expression \\
\hline Sum squares errors & $\sum_{i=1}^{n}\left(X_{\text {cal }}-X_{\text {exp }}\right)_{i}^{2}$ \\
$\begin{array}{l}\text { Mean sum of the per- } \\
\text { cent errors }\end{array}$ & $\frac{100}{n} \sum_{i=1}^{n}\left|\frac{X_{\text {cal }}-X_{\text {exp }}}{X_{\text {exp }}}\right|_{i}$ \\
$\begin{array}{l}\text { Hybrid fractional error } \\
\text { function }\end{array}$ & $\frac{100}{n-p} \sum_{i=1}^{n}\left|\frac{X_{\text {cal }}-X_{\text {exp }}}{X_{\exp }}\right|_{i}$ \\
$\begin{array}{l}\text { Marquardt's percent } \\
\text { standard deviation }\end{array}$ & $100 \sqrt{\frac{1}{n-p}} \sum_{i=1}^{n}\left(\frac{X_{\text {cal }}-X_{\text {exp }}}{X_{\exp }}\right)_{i}^{2}$ \\
\hline
\end{tabular}

$n$ is the number of data point, $p$ is the number of parameter, $X_{\text {cal }}$ is the calculated value of parameter $X$, and $X_{\exp }$ is the measured value of parameter $X$ by the experiment 
are indispensable to solve the IAST model (Myers and Prausnitz, 1965; Radke and Prausnitz, 1972; Lo and Alok, 1996)

$$
\begin{aligned}
& C_{i}=z_{i} C^{*}, \\
& \frac{1}{q_{T}}=\sum_{i}^{N} z_{i} / q_{i}, \\
& q_{i}=z_{i} q, \\
& q_{T}=\sum_{i}^{N} q_{i}, \\
& \sum_{i}^{N} z_{i}=1, \\
& q_{i}=f\left(C_{i}\right),
\end{aligned}
$$

where $z_{i}$ is the molar fraction of species $i$, and $N$ is the number of the species in mixture. Eq. (18) denotes the isotherm of a single-compound solution of solute $i$. Sometimes modification of the IAST model is required to better represent some specific adsorption systems such as solutions containing humic substances (Weber and Smith, 1987). Some other isotherms are shown in Table 2.

\section{Modeling of fixed-bed adsorption}

As one of the most prevalent techniques for separation and purification, fixed-bed adsorption has been widely applied for its high efficiency and easy operation. How to optimize the design and operation conditions of the fixed-bed adsorption is obviously an important issue to be focused on. Given the fact that experimental determination of the adsorption performance under diverse conditions is usually expensive and time-consuming, development of mathematical models to predict fixed-bed adsorption is necessary. An ideal model should be mathematically convenient, be able to give an exact estimation of the breakthrough behavior, and evaluate the effect of each variable on adsorption.

A dynamic adsorption model usually consists of a macroscopic mass conservation equation, uptake rate equation(s) and isotherm. Considering the different components of the adsorption systems (sol-

\begin{tabular}{|c|c|c|}
\hline Expression & Description & Reference \\
\hline$q_{\mathrm{e}, i}=\frac{q_{\mathrm{m}} b_{i} C_{\mathrm{e}, i}}{1+\sum_{i}^{N} b_{i} C_{\mathrm{e}, i}}$ & $\begin{array}{l}\text { Multi-component } \\
\text { Langmuir isotherm }\end{array}$ & (Silva et al., 2010) \\
\hline$q_{\mathrm{e}, i}=\frac{q_{\mathrm{m}} b_{i}\left(C_{\mathrm{e}, i}\right)^{k_{i}}}{1+\sum_{i}^{N} b_{i}\left(C_{\mathrm{e}, i}\right)^{k_{i}}}$ & $\begin{array}{l}\text { Multi-component } \\
\text { Langmuir-Freundlich isotherm }\end{array}$ & (Ruthven, 1984) \\
\hline $\begin{aligned} q_{\mathrm{e}, 1} & =\frac{q_{\mathrm{m}, 2} b_{1} C_{\mathrm{e}, 1}}{1+b_{1} C_{\mathrm{e}, 1}+b_{2} C_{\mathrm{e}, 2}}+\frac{\left(q_{\mathrm{m}, 1}-q_{\mathrm{m}, 2}\right) b_{1} C_{\mathrm{e}, 1}}{1+b_{1} C_{\mathrm{e}, 1}} \\
q_{\mathrm{e} 2} & =\frac{q_{\mathrm{m}, 2} b_{2} C_{\mathrm{e}, 2}}{1+b_{1} C_{\mathrm{e}, 1}+b_{2} C_{\mathrm{e}, 2}}\end{aligned}$ & $\begin{array}{l}\text { Assume the maximum adsorptive } \\
\text { capacity of species } 1 \text { is higher than } \\
\text { species } 2 \text { and the surplus part is } \\
\text { treated as single-component adsorption }\end{array}$ & $\begin{array}{c}\text { (Jain and Snoeyink, } \\
1973 \text { ) }\end{array}$ \\
\hline $\begin{array}{l}q_{\mathrm{e}, 1}=\frac{q_{\mathrm{m}} b_{1} C_{\mathrm{e}, 1}\left[1+\left(K^{\prime} / b_{1}\right) C_{\mathrm{e}, 2}\right]}{1+b_{1} C_{\mathrm{e}, 1}+b_{2} C_{\mathrm{e}, 2}+2 K C_{\mathrm{e}, 1} C_{\mathrm{e}, 2}} \\
q_{\mathrm{e}, 2}=\frac{q_{\mathrm{m}} b_{2} C_{\mathrm{e}, 2}\left[1+\left(K^{\prime} / b_{2}\right) C_{\mathrm{e}, 1}\right]}{1+b_{1} C_{\mathrm{e}, 1}+b_{2} C_{\mathrm{e}, 2}+2 K^{\prime \prime} C_{\mathrm{e}, 1} C_{\mathrm{e}, 2}}\end{array}$ & $\begin{array}{l}\text { Postulate formation of } A d-M_{1}-M_{2} \text {, } \\
\text { Ad is the adsorbent, and } M_{1} \text { and } \\
M_{2} \text { represent species } 1 \text { and } 2 \text {. }\end{array}$ & $\begin{array}{c}\text { (Chong and Volesky, } \\
1995)\end{array}$ \\
\hline $\begin{array}{l}q_{1}=\frac{n_{1} R_{\mathrm{sm}} K_{1} C_{1}^{n_{1}}}{1+K_{1} C_{1}^{n_{1}}+K_{2} C_{2}^{n_{2}}}, \quad q_{2}=\frac{n_{2} R_{\mathrm{sm}} K_{2} C_{2}^{n_{2}}}{1+K_{1} C_{1}^{n_{1}}+K_{2} C_{2}^{n_{2}}}, \\
K_{1}=\frac{\left[R_{\mathrm{s}} M_{1 n_{1}}\right]}{\left[M_{1}\right]^{n_{1}}\left[R_{\mathrm{s}}\right]}, \quad K_{2}=\frac{\left[R_{\mathrm{s}} M_{2 n_{2}}\right]}{\left[M_{2}\right]^{n_{2}}\left[R_{\mathrm{s}}\right]}\end{array}$ & $\begin{array}{l}\text { Assume that adsorption of } \\
\text { species } \mathrm{M}_{1} \text { and } \mathrm{M}_{2} \text { follows } \\
\qquad n_{1} M_{1}+R_{\mathrm{s}} \Leftrightarrow R_{\mathrm{s}} M_{1 n_{1}} \\
n_{2} M_{2}+R_{\mathrm{s}} \Leftrightarrow R_{\mathrm{s}} M_{2 n_{2}}\end{array}$ & (Xue et al., 2009) \\
\hline
\end{tabular}
vents, adsorbate, adsorbent), variable operation conditions and specific demands of accuracy and calculative simplicity, it is an important but challenging task to propose a general use model, because most

Table 2 Lists of some multi-component isotherms 
models derived from different assumptions are only suitable for a limited situation but fail to describe others. In this section, some widely used models are presented and discussed to choose proper models when needed. Note that, toward the solution of unknown composition, a full illustration of modeling was provided elsewhere (Crittenden et al., 1985) and here, we do not intend to discuss that case.

\subsection{General rate models}

Based on the assumption that the rate of intrapellet diffusion is described by Fick's Law, some different expressions of general rate models were developed, such as the pore diffusion model (PDM), homogeneous surface diffusion model (HSDM), and pore and surface diffusion model (PSDM).

PDM can be described as (Du et al., 2008)

$$
\varepsilon \frac{\partial C}{\partial t}+\rho \frac{\partial q}{\partial t}=\frac{\rho D_{\mathrm{ep}}}{r^{2}} \frac{\partial}{\partial r}\left(r^{2} \frac{\partial q}{\partial r}\right)
$$

with the initial and boundary conditions as

$$
\begin{aligned}
& 0 \leq z \leq H, 0 \leq r \leq r_{\mathrm{p}}, t=0 \rightarrow q=0, \\
& r=0 \rightarrow \frac{\partial q}{\partial r}=0, \\
& r=r_{\mathrm{p}} \rightarrow D_{\text {ep }}\left(\frac{\partial q}{\partial r}\right)_{r=r_{\mathrm{p}}}=k_{\mathrm{f}} a\left(C-C_{\mathrm{s}}\right),
\end{aligned}
$$

where $\rho$ is the bed density, $r_{\mathrm{p}}$ is the radius of adsorbent pellets, $D_{\text {ep }}$ refers to the effective pore diffusion coefficient, and $r$ is the distance to the centre of the pellet.

The basic mathematic form of HSDM is (Tien, 1994)

$$
\varepsilon \frac{\partial C}{\partial t}+\rho \frac{\partial q}{\partial t}=\frac{D_{\mathrm{s}}}{r^{2}} \frac{\partial}{\partial r}\left(r^{2} \frac{\partial q}{\partial r}\right)
$$

with its initial and boundary conditions as

$$
\begin{aligned}
& 0 \leq z \leq H, 0 \leq r \leq r_{\mathrm{p}}, t=0 \rightarrow q=0, \\
& r=0 \rightarrow \frac{\partial q}{\partial r}=0, \\
& r=r_{\mathrm{p}} \rightarrow \rho D_{\mathrm{s}}\left(\frac{\partial q}{\partial r}\right)_{r=r_{\mathrm{p}}}=k_{\mathrm{f}} a\left(C-C_{\mathrm{s}}\right),
\end{aligned}
$$

where $D_{\mathrm{s}}$ is the surface diffusion coefficient.

In addition, PSDM can be represented as (Liu et al., 2010)

$$
\varepsilon \frac{\partial C}{\partial t}+\rho \frac{\partial q}{\partial t}=\frac{D_{\mathrm{s}}}{r^{2}} \frac{\partial}{\partial r}\left(r^{2} \frac{\partial q}{\partial r}\right)+\frac{\rho D_{\mathrm{ep}}}{r^{2}}\left(r^{2} \frac{\partial q}{\partial r}\right)
$$

with its specific initial and boundary conditions of

$$
\begin{aligned}
& 0 \leq z \leq H, 0 \leq r \leq r_{\mathrm{p}}, t=0 \rightarrow q=0, \\
& r=0 \rightarrow \frac{\partial q}{\partial r}=0, \\
& r=r_{\mathrm{p}} \rightarrow\left[\left(D_{\mathrm{ep}}+D_{\mathrm{s}} \rho \frac{\mathrm{d} q}{\mathrm{~d} C}\right) \frac{\partial C}{\partial r}\right]_{r=r_{\mathrm{p}}}=k_{\mathrm{f}} a\left(C-C_{\mathrm{s}}\right) .
\end{aligned}
$$

Due to the fact that $\rho \frac{\partial q}{\partial t}$ is usually much higher than $\varepsilon \frac{\partial C}{\partial t}$, the latter term was neglected in most cases.

With distinct rate control step(s) of different systems, the appropriate type of the general rate models should be applied, including the film-pore diffusion model, film-surface diffusion model and film-pore/surface diffusion model. Reasonably, the film diffusion can be neglected when the film mass transfer resistance is quite small (i.e., negligible concentration gradient in the film). However, additional experiments should be performed to ensure that film diffusion is not a rate control step. Note that among Eqs. (19)-(21), the term of the surface reaction rate is not involved in most cases because it is much faster than other diffusion steps. Sometimes the surface reaction should be considered when it significantly affects the total adsorption rate or even becomes the sole rate control step. Plazinski et al. (2009) made a comprehensive review of sorption kinetics including surface reaction mechanism.

When a proper liquid phase continuity equation (Eq. (1) or (2)), film diffusion equation (Eq. (3)), intrapellet diffusion equation, and isotherm equation are available, it is possible to generate the breakthrough curve by solving these partial differential equations. Note that several parameters in the general rate models can be determined both theoretically and experimentally. The theoretical method is based on some basic correlations (Roberts et al., 1985; 
Crittenden et al., 1986; Weber and Smith, 1987; Wolborska, 1989b; Tien, 1994; Sperlich et al., 2005; Worch, 2008) and part of them are listed in Table 3, whereas the accuracy of estimation values of the parameters is not satisfactory. Especially, to our knowledge, there is no reliable method to theoretically predict the tortuosity $\tau$ and surface diffusion coefficient $D_{\text {s }}$, which are indispensable when using the general rate models. Thus, for trustworthy prediction, it is inevitable to use the experimental method to determinate these parameters.
To directly solve the general rate models is usually a time-consuming and computationally troubled work. Some convenient methods such as the finite difference method and orthogonal collocation method (Mathews and Weber, 1977; Kaczmarski and Antos, 1996; McKay, 2001; Finlayson, 2003; Lee and McKay, 2004) were developed to obtain the numerical solution with the aid of several computational softwares.

Except for PDM, HSDM and PSDM, some other models based on Fick's law were also available and summarized in Table 4

Table 3 Some basic correlations to determine the parameters of the general rate models

\begin{tabular}{|c|c|c|c|}
\hline Parameter & Correlation & Condition & Reference \\
\hline \multirow[t]{4}{*}{$\begin{array}{l}\text { Bulk liquid diffusivity } \\
\text { of the adsorbate, } D_{\mathrm{M}}\end{array}$} & $D_{\mathrm{M}} \eta / T=7.4 \times 10^{-8}(\beta M)^{0.5} / V_{\mathrm{m}}^{0.6}$ & - & $\begin{array}{c}\text { (Wilke and Chang, } \\
1955)\end{array}$ \\
\hline & $0.01498 T^{1.81} \sqrt{\frac{1}{M}+\frac{1}{M}}$ & - & $\begin{array}{l}\text { (Puértolas et al., } \\
\text { 2010) }\end{array}$ \\
\hline & $D_{\mathrm{M}}=\frac{\sqrt{M_{i}} M_{j}}{P\left(T_{C_{i}} T_{C_{j}}\right)^{0.1405}\left(V_{m_{i}}^{0.4} V_{m_{j}}^{0.4}\right)^{2}}$ & & \\
\hline & $D_{\mathrm{M}}=\frac{3.595 \times 10^{-14} T}{\eta M^{0.53}}$ & - & (Worch, 1993) \\
\hline \multirow[t]{13}{*}{$\begin{array}{l}\text { Film (external) diffu- } \\
\text { sivity, } k_{\mathrm{f}}\end{array}$} & $S h=\frac{1.1}{\varepsilon} P e^{1 / 3}$ & - & (Tan et al., 1975) \\
\hline & $S h=\frac{1.09}{\varepsilon} P \mathrm{e}^{1 / 3}$ & $\begin{array}{c}0.0016<\varepsilon R e<55 \\
950<S_{c}<70000\end{array}$ & $\begin{array}{c}\text { (Wilson and } \\
\text { Geankoplis, 1966) }\end{array}$ \\
\hline & $S h=2+1.58 R e^{0.4} S c^{1 / 3}$ & $0.001<\operatorname{Re}<5.8$ & (Ohashi et al., 1981) \\
\hline & $S h=2+1.21 R e^{0.5} S c^{1 / 3}$ & $5.8<R e<500$ & \\
\hline & $S h=2+0.59 R e^{0.6} S c^{1 / 3}$ & $R e>500$ & \\
\hline & $S h=2.4 \varepsilon R e^{0.3} S c^{0.42}$ & $\begin{array}{l}0.08<R e<125 \\
150<S_{c}<1300\end{array}$ & $\begin{array}{c}\text { (Williamson et al., } \\
\text { 1963) }\end{array}$ \\
\hline & $S h=\frac{0.325}{\varepsilon R e^{0.36} S c^{1 / 3}}$ & - & (Ko et al., 2003) \\
\hline & $S h=2+1.1 R e^{0.6} S c^{1 / 3}$ & $3.0<\operatorname{Re}<10000$ & $\begin{array}{l}\text { (Wakao and } \\
\text { Funazkri, 1978) }\end{array}$ \\
\hline & $S h=1.85[(1-\varepsilon) / \varepsilon]^{1 / 3} \operatorname{Re}^{1 / 3} S c^{1 / 3}$ & $\operatorname{Re}[\varepsilon /(1-\varepsilon)]<100$ & $\begin{array}{c}\text { (Kataoka et al., } \\
1972 \text { ) }\end{array}$ \\
\hline & $S h=\left(2+0.644 R e^{1 / 2} S c^{1 / 3}\right)[1+1.5(1-\varepsilon)]$ & - & $\begin{array}{l}\text { (Chern and Chien, } \\
\text { 2002) }\end{array}$ \\
\hline & $S h=\left[2+\left(S h_{\mathrm{L}}^{2}+S h_{\mathrm{T}}^{2}\right)^{0.5}\right][1+1.5(1-\varepsilon)]$ & $\begin{array}{l}R e S c>500 \\
S c<12000\end{array}$ & (Gnielinski, 1978) \\
\hline & $S h_{\mathrm{L}}=0.644 R e^{1 / 2} S c^{1 / 3}$ & & \\
\hline & $S h_{\mathrm{T}}=\left(0.037 R e^{0.8} S c\right) /\left[1+2.443 \operatorname{Re}^{-0.1}\left(S c^{2 / 3}-1\right)\right]$ & & \\
\hline $\begin{array}{l}\text { Knudsen diffusion } \\
\text { coefficient, } D_{\mathrm{k}}\end{array}$ & $D_{\mathrm{k}}=9700 r_{\mathrm{ap}} \sqrt{\frac{T}{M}}$ & - & $\begin{array}{l}\text { (Scott and Dullien, } \\
1962)\end{array}$ \\
\hline $\begin{array}{l}\text { Pore diffusion coeffi- } \\
\text { cient, } D_{\mathrm{p}}\end{array}$ & $D_{\mathrm{p}}=\varepsilon D_{\mathrm{M}} / \tau$ & - & - \\
\hline $\begin{array}{l}\text { Effective pore diffu- } \\
\text { sion coefficient, } D_{\text {ep }}\end{array}$ & $\frac{1}{D_{\mathrm{ep}}}=\frac{1}{D_{\mathrm{p}}}+\frac{1}{D_{\mathrm{k}}}$ & - & - \\
\hline
\end{tabular}

$\eta$ is the dynamic viscosity, $r_{\text {ap }}$ is the average pore radius of adsorbent, $M$ is the molecular weight, $\beta$ is the association constant of the solvent and $\beta=2.6$ for water and 1.5 for ethanol, $V_{\mathrm{m}}$ is the molar volume of solute at normal boiling point, and $T_{\mathrm{c}}$ is the critical temperature 
Table 4 Lists of some general rate type models

\begin{tabular}{lc}
\hline \multicolumn{1}{c}{ Modification } & Assumption \\
\hline Macropore diffu- & Postulating each adsorbent \\
sion and micro- & pellet is composed of a core \\
pore diffusion (in with micropore $\left(0<r<r_{\mathrm{c}}\right)$ and \\
series) & $\begin{array}{l}\text { outer region with macropore } \\
\left(r_{\mathrm{c}}<r<r_{\mathrm{p}}\right)\end{array}$
\end{tabular}

Equation
Diffusion equation of macropore region:
$\frac{\partial q}{\partial t}=\frac{1}{r^{2}} \frac{\partial}{\partial r}\left[\left(D_{\mathrm{e}}\right)_{\mathrm{Ma}} r^{2} \frac{\partial q}{\partial r}\right], r_{\mathrm{c}} \leq r \leq r_{\mathrm{p}}$
Diffusion equation of micropore region:

$\frac{\partial q}{\partial t}=\frac{1}{r^{2}} \frac{\partial}{\partial r}\left[\left(D_{\mathrm{e}}\right)_{\mathrm{mi}} r^{2} \frac{\partial q}{\partial r}\right], 0 \leq r \leq r_{\mathrm{c}} ;$

Mass conservation at the interface:

$\left(D_{\mathrm{e}}\right)_{\mathrm{Ma}}\left(\frac{\partial q}{\partial r}\right)_{r \rightarrow r_{\mathrm{c}}^{+}}=\left(D_{\mathrm{e}}\right)_{\mathrm{mi}}\left(\frac{\partial q}{\partial r}\right)_{r \rightarrow r_{\mathrm{c}}^{-}}$

Macropore diffu- The so-called branched pore sion and micro- kinetic model applies to adpore diffusion (in sorption processes controlled parallel) by the pore-surface diffusion. Divide the adsorbent pellet into two regions, micropore and macropore region, and assume both of them array parallel

Diffusion equation of macropore:

$f \frac{\partial q_{\mathrm{Ma}}}{\partial t}=f \frac{1}{r^{2}} \frac{\partial}{\partial r}\left[\left(D_{\mathrm{e}}\right)_{\mathrm{Ma}} r^{2} \frac{\partial q_{\mathrm{Ma}}}{\partial r}\right]-R_{\mathrm{b}}$

Diffusion equation of micropore:

$(1-f) \frac{\partial q_{\mathrm{mi}}}{\partial t}=(1-f) \frac{1}{r^{2}} \frac{\partial}{\partial r}\left[\left(D_{\mathrm{e}}\right)_{\mathrm{mi}} r^{2} \frac{\partial q_{\mathrm{mi}}}{\partial r}\right]+R_{\mathrm{b}}$;

Total adsorption amount:

$q=f q_{\mathrm{Ma}}+(1-f) q_{\mathrm{mi}}$

Transfer rate of solute from macropore to micropore:

$R_{\mathrm{b}}=k_{\mathrm{b}}\left(q_{\mathrm{Ma}}-q_{\mathrm{mi}}\right)$

Shrinking core This model used to describe theory model the intrapellet adsorption is controlled by pore diffusion, supposing adsorption firstly occurs at the outer region of adsorbent, then the mass transfer zone moves inward together with the extending of saturated outer region and shrinking of unloaded core (Fig. 5)

The mass transfer flux
$J=4 \pi r_{\mathrm{p}}^{2} k_{\mathrm{f}}\left(C-C_{\mathrm{e}}\right) ;$

Diffusion in the pore of adsorbent (Fick's Law):

$J=\frac{4 \pi C_{\mathrm{e}} r_{\mathrm{p}} r_{\mathrm{c}} D_{\mathrm{ep}}}{r_{\mathrm{p}}-r_{\mathrm{c}}}$

(Peel et al., 1981; Yang and Al-Duri, 2001; Ko et al., 2002)

The velocity of the mass transfer zone:

$\frac{\mathrm{d} r_{\mathrm{c}}}{\mathrm{d} t}=-\frac{J}{4 \pi r_{\mathrm{c}}^{2} \rho q_{\mathrm{e}}}$;

The mean concentration of adsorbed solute:

$\bar{q}=q_{\mathrm{e}}\left[1-\left(r_{\mathrm{c}} / r_{\mathrm{p}}\right)^{3}\right]$

HSDM involved When additional reaction additional reac- occurs in adsorption process, tion the conventional HSDM considering only the diffusion mechanism is no longer suitable. For this matter, modification were made to meet the demand

Diffusion and reaction combined equation:

(Abuzaid and

$\frac{\partial q}{\partial t}=\frac{1}{r^{2}} \frac{\partial}{\partial r}\left(D_{\mathrm{s}} r^{2} \frac{\partial q}{\partial r}\right)-R_{\mathrm{r}}$

Nakhla, 1997)

For example, when $R_{\mathrm{r}}$ is expressed by

$R_{\mathrm{r}}=K_{\mathrm{r}}\left(q-q_{\mathrm{e}}\right)$;

The diffusion-reaction rate equation is written as

$\frac{\partial q}{\partial t}=\frac{1}{r^{2}} \frac{\partial}{\partial r}\left(D_{\mathrm{s}} r^{2} \frac{\partial q}{\partial r}\right)-K_{\mathrm{r}}\left(q-q_{\mathrm{e}}\right)$

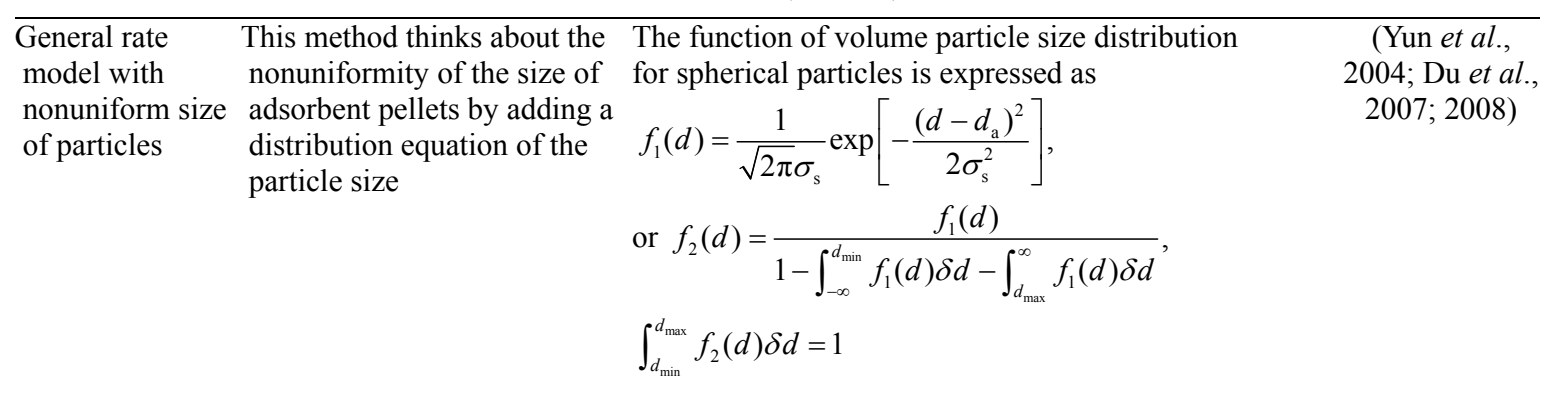

2001; Quek and Al-Duri, 2007; Traylor et al., 2011) 
(Table 4)

\begin{tabular}{|c|c|c|c|}
\hline $\begin{array}{l}\text { General rate } \\
\text { model with } \\
\text { concentration- } \\
\text { dependent sur- } \\
\text { face diffusion } \\
\text { coefficient }\end{array}$ & $\begin{array}{l}\text { To describe the change of surface } \\
\text { diffusion coefficient under dif- } \\
\text { ferent coverage, functions of } \\
D_{\mathrm{s}}(q) \text { were proposed. In case } 3 \text {, } \\
\text { according to (Cussler, 1976), the } \\
\text { cross-term Fickian diffusivities, } \\
D_{12} \text { and } D_{21} \text { are usually neglected } \\
\text { because they are generally less } \\
\text { than } 10 \% \text { of } D_{11} \text { and } D_{22}\end{array}$ & $\begin{array}{l}\text { 1. } D_{\mathrm{s}}(q)=D_{\mathrm{s} 0} \exp \left[k_{\mathrm{s}}\left(\frac{q}{q_{\mathrm{m}}}\right)\right] ; \\
\text { 2. } D_{s}(q)=D_{\mathrm{s} 0} \frac{\partial \ln C}{\partial \ln q} ; \\
\text { 3. For binary system, } \\
\qquad \frac{\partial q_{i}}{\partial t}=\frac{1}{r^{2}} \frac{\partial}{\partial r}\left[r^{2}\left(D_{\mathrm{s}, i i} \frac{\partial q_{i}}{\partial r}+D_{\mathrm{s}, i j} \frac{\partial q_{j}}{\partial r}\right)\right] \text {, } \\
\qquad D_{\mathrm{s}, i j}(q)=D_{\mathrm{s} 0, i}\left(\frac{q_{i}}{q_{j}}\right) \frac{\partial C_{i}}{C_{i}} / \frac{\partial q_{j}}{q_{j}}\end{array}$ & $\begin{array}{l}\text { (Neretnieks, } \\
\text { 1976; Ko et al., } \\
\text { 2005; Lee et al., } \\
\text { 2005; Jia and } \\
\text { Lua, 2008) }\end{array}$ \\
\hline
\end{tabular}

$\left(D_{\mathrm{e}}\right)_{\mathrm{Ma}}$ : effective diffusion coefficient of marcopore region; $\left(D_{\mathrm{e}}\right)_{\mathrm{mi}}$ : effective diffusion coefficient of micropore region; $R_{\mathrm{b}}$ : branched pore kinetic model rate constant; $q_{\mathrm{Ma}}$ : adsorbed concentration of the macropore zone; $q_{\mathrm{mi}}$ : adsorbed concentration of the micropore zone; $f$ : volume fraction of the macropore region; $K_{\mathrm{r}}$ : reaction rate coefficient; $k_{\mathrm{b}}$ : model kinetic coefficient; $k_{\mathrm{s}}$ : model constant; $r_{\mathrm{c}}$ : critical radius; $\sigma_{\mathrm{s}}$ : standard deviation of the particles; $d$ : diameter of the adsorbent pellets; $d_{\mathrm{a}}$ : average diameter of the adsorbent pellets; $D_{\mathrm{s} 0}$ : surface diffusion coefficient when the coverage is zero (self-diffusivity)

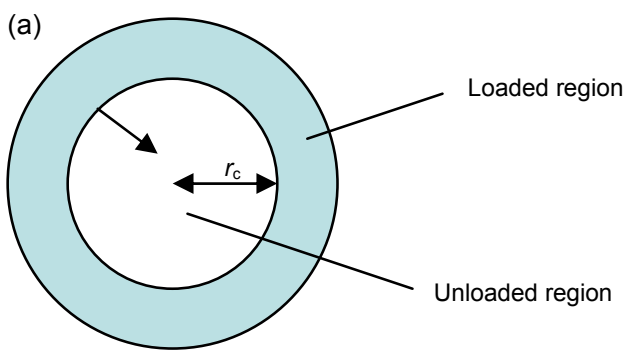

(b)

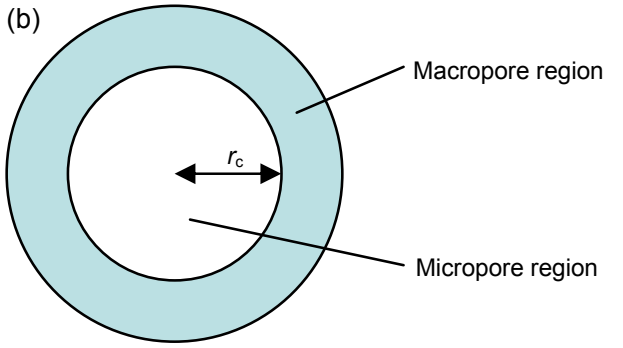

Fig. 5 Schematic diagram of branched pore kinetic model (a) and shrinking core theory model (b)

A review of surface diffusion is available elsewhere (Medved and Cerny, 2011), which might provide some ideas to modify the intrapellet diffusion models.

The prevalence of the conventional/modified general rate models is not only because they give good prediction of dynamic adsorption, but also involves a variety of parameters to determine the process variables. The variables could be optimized by keeping other parameters constant and comparing the breakthrough curves predicted by this model under different values of the target parameter " $X$ ", for example, $0.5 X, X$ and $2 X$ (Note that adjusting a parameter may lead to variation in the phenomenological coefficient(s), and optimization based on modeling is not a precise method). However, the main limitation of these models is the complicated and time-consuming computation.

\subsection{Linear driving force (LDF) model}

The linear driving force (LDF) model proposed by (Glueckauf, 1955) formulates a lumped mass transfer coefficient to represent the intrapellet diffusion rate, as written in a linear form as

$$
\frac{\mathrm{d} q}{\mathrm{~d} t}=k_{\mathrm{e}}\left(q_{\mathrm{s}}-q_{\mathrm{a}}\right)
$$

where $q_{\mathrm{a}}$ is the average concentration of the adsorbed adsorbate, $q_{\mathrm{s}}$ is the loading of the adsorbate at the external surface of adsorbent, and $k_{\mathrm{e}}$ is the lump intrapellet diffusion kinetic coefficient. The original LDF model regards that $q_{\mathrm{s}}$ in equilibrium corresponds to the concentration of the bulk solution $C$. In other words, it neglected the concentration gradient in the interface region between the liquid phase and adsorbent, and $k_{\mathrm{e}}$ is a function of intrapellet diffusivity. When the mass transfer within the interface is involved, the film diffusion equation should be added into the LDF model, namely

$$
\frac{\mathrm{d} q}{\mathrm{~d} t}=k_{\mathrm{f}} a\left(C-C_{\mathrm{s}}\right) .
$$


Combining Eqs. (22) and (23) with the liquid phase mass conservation equation and proper isotherm, it is possible to predict the breakthrough curve. Hence, the LDF model can be used to estimate the coefficients $k_{\mathrm{e}}$ and $k_{\mathrm{f}}$. Similar to the general rate models, these parameters can be acquired by the theoretical and experimental ways. For the theoretical way, a widely accepted expression is written as (Glueckauf, 1955)

$$
k_{\mathrm{e}}=\frac{15 D_{\mathrm{e}}}{r_{\mathrm{p}}^{2}}
$$

When intrapellet diffusion is dominated by pore diffusion

$$
k_{\mathrm{e}}=\frac{15 D_{\mathrm{p}}}{f^{\prime}(C) \rho r_{\mathrm{p}}^{2}} .
$$

While by surface diffusion

$$
k_{\mathrm{e}}=\frac{15 D_{\mathrm{s}}}{r_{\mathrm{p}}^{2}} .
$$

An empirical equation of the rate constant was developed previously (Heese and Worch, 1997; Worch, 2008)

$$
k_{\mathrm{e}}=0.00129\left[D_{\mathrm{M}} C_{\mathrm{F}} /\left(r_{\mathrm{p}}^{2} q_{\mathrm{F}}\right)\right]^{1 / 2},
$$

where $D_{\mathrm{e}}$ is the effective intrapellet diffusion coefficient involving both surface diffusion and pore diffusion, $f^{\prime}(C)$ is related to the isotherm. Except for $D_{\mathrm{s}}$ and $D_{\mathrm{e}}$, all the parameters are available by directly measuring or referring to the corresponding isotherms. Note that $\frac{\mathrm{d} q}{\mathrm{~d} c}$ is not constant if the isotherm is nonlinear (i.e., does not obey Henry's Law). Hence, to obtain the value of $D_{\mathrm{e}}$, an average value has to be applied to represent $f^{\prime}(\mathrm{C})$. As for $D_{\mathrm{s}}$, it can be reckoned by combining Eqs. (26) and (27):

$$
\frac{15 D_{\mathrm{s}}}{r_{\mathrm{p}}^{2}}=0.00129\left[D_{\mathrm{M}} C_{\mathrm{F}} /\left(r_{\mathrm{p}}^{2} q_{\mathrm{F}}\right)\right]^{1 / 2},
$$

and then calculate $D_{\mathrm{e}}$ by

$$
D_{\mathrm{e}}=D_{\mathrm{s}}+D_{\mathrm{p}} /\left(f^{\prime}(C) \rho\right)
$$

The calculated kinetic parameters sometimes fit well with the experimental data, but conspicuous deviation may appear in other cases. For instance, the theoretical value is often much higher than the experimental result when the dissolved organic matter (DOM) is adsorbed. In that case, the theoretical method could just approximately reckon the adsorption performance, and the parameters have to be determined by the experimental breakthrough curves.

Another expression of the LDF model is available by incorporating Eqs. (22) and (23)

$$
\frac{\partial q}{\partial t}=k_{\mathrm{o}}\left(q_{\mathrm{s}}-q_{\mathrm{a}}\right),
$$

where $k_{\mathrm{o}}$ is the overall kinetic constant combining both the film diffusivity and the intrapellet diffusivity. Both expressions of the LDF model have widespread application (Murillo et al., 2004; Borba et al., 2006; Puértolas et al., 2010; Silva et al., 2010). Additionally, surface reaction was also involved in LDF in some cases (Szukiewicz, 2000; 2002). Computational software is often required to derive the numerical solution of the LDF model, especially when the isotherm is in the nonlinear form. Nevertheless, as compared to the general rate models, the LDF model could reduce the computational time significantly and its accuracy was generally acceptable. Hence, the LDF model has become one of the most widely used models now. For some recent developments of the LDF model one could refer to the work by Gholami and Talaie (2010). The limitations of the LDF model were also discussed elsewhere (Nakao and Suzuki, 1983; Do and Mayfield, 1987; Yao and Tien, 1993; Zhang and Ritter, 1997), such as the limited applicability of the conventional expression of $k_{\mathrm{e}}$ in many systems.

\subsection{Wave propagation theory}

A novel method to evaluate the service volume (or service time) was proposed by (Helfferich and Klein, 1970). They defined the wave velocity as the velocity of a given value of a variable and made an analog to explain the concept of wave (Helfferich and 
Carr, 1993), from which one can easily deduce the "concentration velocity" as

$$
u_{C}=\left(\frac{\partial z}{\partial t}\right)_{C}=-\frac{\left(\frac{\partial C}{\partial t}\right)_{z}}{\left(\frac{\partial C}{\partial z}\right)_{t}},
$$

where $u_{C}$ is the velocity of the "concentration wave". The liquid phase continuity equation applied in the wave propagation theory can be expressed as

$$
\rho \frac{\partial q}{\partial t}+\varepsilon \frac{\partial C}{\partial t}+u_{i} \varepsilon \frac{\partial C}{\partial z}=0
$$

where $u_{i}$ is the interstitial velocity. Combining Eqs. (31) and (32), we can obtain:

$$
u_{C}=\frac{u_{i}}{1+\frac{\rho}{\varepsilon}\left(\frac{\mathrm{d} q}{\mathrm{~d} C}\right)} .
$$

Assuming the adsorption rate is infinite (i.e., Local equilibrium), $\frac{\mathrm{d} q}{\mathrm{~d} C}$ is available to be calculated by the corresponding isotherm

$$
q_{\mathrm{e}}=f\left(C_{\mathrm{e}}\right)
$$

Especially, for a self-sharpening wave, the wave velocity is calculated by

$$
u_{C}=\frac{u_{i}}{1+\frac{\rho}{\varepsilon}\left(\frac{\Delta q}{\Delta C}\right)} .
$$

For better understanding of the terminologies "self-sharpening" and "non-sharpening", one can refer to some previous works where the chromatographic phenomena were discussed in detail (Helfferich and Klein, 1970; Helfferich, 1984; Helfferich and Carr, 1993; Helfferich and Whitley, 1996; Chern and Huang, 1999). From the wave velocity the breakthrough volume (or service time) can be calculated, for the self-sharpening wave (Chern and Huang, 1999)

$$
\frac{V_{\mathrm{bk}}}{V_{\mathrm{b}}}=\varepsilon\left(1+\frac{\rho}{\varepsilon} \frac{\Delta q}{\Delta C}\right)=\varepsilon\left(1+\frac{\rho}{\varepsilon} \frac{q_{\mathrm{F}}-q}{C_{\mathrm{F}}-C}\right)
$$

for non-sharpening wave

$$
\frac{V_{\mathrm{bk}}}{V_{\mathrm{b}}}=\varepsilon\left(1+\frac{\rho}{\varepsilon} \frac{\mathrm{d} q}{\mathrm{~d} C}\right)
$$

where $V_{\mathrm{bk}}$ is the breakthrough volume, and $V_{\mathrm{b}}$ is the bed volume. Apparently, this ingenious method brings considerably mathematical convenience to predict an adsorption system and the only information needed is the isotherm. Once the isotherm information $\left(\frac{\mathrm{d} q}{\mathrm{~d} t}\right.$ or $\left.\Delta q / \Delta t\right)$ is available, the service time can be directly calculated by Eq. (36) or Eq. (37). On the other hand, the hypothesis of the infinite mass transfer velocity is too arbitrary when the flow rate is not so slow, and the results calculated from the wave theory may deviate from the experimental data notably. Moreover, when the molecular diffusion cannot be neglected or Eq. (2) is used as the liquid phase continuity equation, the service time cannot be acquired by the wave velocity.

\subsection{Constant pattern theory}

Even though the wave propagation theory has limited range of application due to the harsh premise, wave propagation is still an interesting concept in modeling. Luckily, the constant pattern theory including the "wave" velocity offers another approach to predict the breakthrough curve. When one assumes that a self-sharpening wave moves at a constant rate in column, $\tau$, the adjusted time, can be represented as (Chern and Chien, 2002; Pan et al., 2005)

$$
\tau=t-\frac{z}{u_{C}}
$$

The liquid phase continuity equation is

$$
\rho \frac{\partial q}{\partial t}+\varepsilon \frac{\partial C}{\partial t}+u_{i} \varepsilon \frac{\partial C}{\partial z}=0
$$

Substituting Eq. (38) into Eq. (39), we can obtain 


$$
\left(1-\frac{u_{i}}{u_{C}}\right) C+\frac{\rho}{\varepsilon} q=0
$$

Thus, an important relationship can be deduced

$$
\frac{C_{1}}{C_{2}}=\frac{q_{1}}{q_{2}}
$$

or

$$
\frac{C}{C_{\mathrm{F}}}=\frac{q}{q_{\mathrm{F}}} .
$$

Combining with the isotherm $q_{\mathrm{e}}=f\left(C_{\mathrm{e}}\right)$ leads to

$$
C_{\mathrm{e}}=f^{-1}\left(C q_{\mathrm{F}} / C_{\mathrm{F}}\right)
$$

The overall mass transfer equation can be expressed as

$$
\rho \frac{\mathrm{d} q}{\mathrm{~d} \tau}=\varepsilon K_{\mathrm{L}} a\left(C-C_{\mathrm{e}}\right)
$$

where $K_{\mathrm{L}}$ is the overall kinetic constant, and $a=3(1-\varepsilon) / r_{\mathrm{p}}$. After integrating Eq. (43) with Eq. (44) and rearranging, we obtain

$$
t=t_{1 / 2}+\frac{\rho q_{\mathrm{F}}}{\varepsilon K_{\mathrm{L}} a C_{\mathrm{F}}} \int_{C_{\mathrm{F} / 2}}^{C} \frac{1}{C-f^{-1}\left(q_{\mathrm{F}} C / C_{\mathrm{F}}\right)} \mathrm{d} C,
$$

where $t_{1 / 2}$ is the time when the concentration of adsorbate in the effluent reaches half of that in the feeding solution, $q_{\mathrm{F}}$ is the value of $q$ in equilibrium with $C_{\mathrm{F}}$. Combining Eq. (45) with a specific isotherm, the breakthrough curve can be generated, for example, with the Langmuir isotherm

$$
t=t_{1 / 2}+\frac{\rho q_{\mathrm{m}}}{\varepsilon K_{\mathrm{L}} a C_{\mathrm{F}}}\left[\ln 2 x+\frac{1}{1+b C_{\mathrm{F}}} \ln \frac{1}{2(1-x)}\right],
$$

or with the Freundlich isotherm

$$
t=t_{1 / 2}+\frac{\rho K C_{\mathrm{F}}^{n-1}}{\varepsilon K_{\mathrm{L}} a} \int_{1 / 2}^{x} \frac{1}{x-x^{1 / n}} \mathrm{~d} x .
$$

Rewriting

$$
\left(\frac{\mathrm{d} x}{\mathrm{~d} t}\right)_{x=0.5}=\frac{\varepsilon K_{\mathrm{L}} a}{\rho K C_{\mathrm{F}}^{n-1}}\left(x-x^{1 / n}\right),
$$

where $x=C / C_{\mathrm{F}}$. In some cases the constant pattern model works in a transcendental way. This is because, when film diffusion is the only rate control step of adsorption, the value of $K_{\mathrm{L}}$ is close to the external diffusion coefficient $k_{\mathrm{f}}$, which could be estimated by the corresponding correlation listed in Table 4. Unfortunately, adsorbent of microporous structure usually has great intrapellet resistance, leading to the limited application of the method. Chern et al. (2002) related the volumetric film diffusion mass transfer coefficient to the volumetric flow rate $Q$

$$
k_{\mathrm{f}} a=k_{1}+k_{2} Q^{1 / 2} .
$$

Kananpanah et al. (2009) also proposed that

$$
\begin{aligned}
& k_{\mathrm{f}} a=k_{1}+k_{2} Q^{n^{\prime}}, \\
& k_{\mathrm{f}} a=k_{1} \mathrm{e}^{k_{2} Q}, \\
& k_{\mathrm{f}} a=k_{1}+k_{2} \mathrm{e}^{k_{3} Q},
\end{aligned}
$$

where specific parameters $k_{1}, k_{2}, k_{3}$ and $n^{\prime}$ can be acquired by fitting the experimental data. Eqs. (49) -(52) pave a way to predict the performance under different volumetric flow rates, but it is not suitable to predict the performance of the adsorption systems with no experimental data. The main functions of the constant pattern theory are: (1) to determine valuable information such as $\rho, \varepsilon, q_{\mathrm{F}}, C_{\mathrm{F}}$, and isotherm; (2) to read $t_{1 / 2}$ through dynamic adsorption; and, (3) to determinate $K_{\mathrm{L}} a$ by proper methods. For instance, when the isotherm follows the Freundlich model, according to Eq. (48), $K_{\mathrm{L}} a$ can be obtained from the tangent slope of $x$ vs. $t$ at $x=1 / 2$. When the Langmuir isotherm is better to describe the equilibrium relationship, $K_{\mathrm{L}} a$ can be calculated by the slope of the plot of $\ln 2 x+\left[1 /\left(1+b C_{\mathrm{F}}\right)\right] \ln [1 /(2-2 x)]$ vs. $t$ (Eq. (46)); and, (4) to generate the complete breakthrough curve by integrating $K_{\mathrm{L}} a$ into a corresponding equation. The constant pattern model gives outstanding prediction of the breakthrough curve in numerous studies. Yet, when the adsorption rate is dominated by the intrapellet diffusion, prediction by this model sometimes deviates from the real situation. 


\subsection{Clark model}

As is well known, granular active carbon (GAC) is one of the most popular adsorbents with micropore structure. US EPA even initiated a series of field studies to evaluate the performance of GAC and acquired sufficient data. Based on the data from US EPA, Clark (1987) developed a model to predict the performance of GAC-organic compounds adsorption system. The Clark model was deduced based on the following equations and assumptions (Clark, 1987).

(1) Liquid phase continuity equation is

$$
J=\frac{Q_{\mathrm{A}} A C-Q_{\mathrm{A}} A(C-\Delta C)}{A \Delta z} .
$$

(2) The shape of the mass-transfer zone is constant and all the adsorbates are removed at the end of the column,

$$
Q_{\mathrm{A}} C=u_{\mathrm{a}} q_{\mathrm{F}} .
$$

(3) The isotherm fits the Freundlich type:

$$
q_{\mathrm{e}}=K C^{1 / n} .
$$

(4) Expression of the adsorption rate is

$$
J=Q_{\mathrm{A}} \frac{\mathrm{d} C}{\mathrm{~d} z}=K_{\mathrm{T}}\left(C-C_{\mathrm{e}}\right),
$$

where $J$ is the mass-transfer rate per unit reactor volume, $A$ is the column cross-section area, $Q_{\mathrm{A}}$ is the volumetric flow rate per unit of cross-section area, $u_{\mathrm{a}}$ is the mass velocity of the adsorbent to maintain the mass-transfer zone stationary, $\Delta C$ is the incremental change of concentration, and $\Delta z$ is the differential reactor height.

The final expression of Clark model is

$$
\begin{aligned}
& \left(\frac{C_{i n}^{n-1}}{1+B \mathrm{e}^{-r^{\prime} t}}\right)^{1 /(n-1)}=C, \\
& B=\left(\frac{C_{i n}^{n-1}}{C_{\mathrm{b}}^{n-1}}-1\right) \mathrm{e}^{r^{\prime} t_{\mathrm{b}}}, \\
& r^{\prime}=(n-1) \frac{K_{\mathrm{T}}}{Q_{\mathrm{a}}} \frac{\mathrm{d} z}{\mathrm{~d} t},
\end{aligned}
$$

where $C_{\mathrm{b}}$ is the breakthrough concentration, $t_{\mathrm{b}}$ is the service time, and $C_{\text {in }}$ is the constant influent value on the carbon bed. The following procedures should be completed prior to using the model.

(1) Determine $n$ by the batch experiment;

(2) Rearrange Eq. (58) into

$$
\ln \left[\left(\frac{C_{\mathrm{F}}}{C}\right)^{n-1}-1\right]=-r^{\prime} t+\ln B .
$$

(3) The slope and intercept of the plot $\ln \left[\left(C_{\mathrm{F}} / C\right)^{1 / n}-1\right]$ vs. $t$ allow one to solve $r$, and $B$, respectively;

(4) Generate the whole breakthrough curve.

It is clear that the mass conservation equation in the column is different from those discussed above. We find that Eq. (53) actually disregards the accumulation of adsorbate concentration. In other words, it does not reflect the time difference between $C$ and $C+\Delta C$. If taking a controlled volume $V$ (the volume passing through any cross-section per unit time) into consideration and postulating the uniformity of the control volume and negligible molecular diffusion, we obtain

$$
\varepsilon V C_{z, t}-\rho V J \Delta t=\varepsilon V C_{z+\Delta z, t+\Delta t} .
$$

When $\Delta t \rightarrow 0$, then

$$
\varepsilon V C_{z, t}-\rho V J \mathrm{~d} t=\varepsilon V C_{z+\mathrm{d} z, t+\mathrm{d} t}
$$

Additionally

$$
C_{z+\mathrm{d} z, t+\mathrm{d} t}=C_{z, t}+\frac{\partial C}{\partial z} \mathrm{~d} z+\frac{\partial C}{\partial t} \mathrm{~d} t .
$$

Cancelling $V \mathrm{~d} t$ of Eq. (62) and combining Eq. (63)

$$
\rho J=-u_{i} \varepsilon \frac{\partial C}{\partial z}-\varepsilon \frac{\partial C}{\partial t} .
$$

Obviously, Eq. (64) is the same with Eq. (32), while deleting the term of $\mathrm{d} t \frac{\partial C}{\partial t}$ will lead to Eq. (53). The same conclusion can also be made when 
assuming $\quad \rho_{\mathrm{a}}(1-\varepsilon)\left(\frac{\partial q}{\partial t}\right)+u\left(\frac{\partial C}{\partial z}\right)>>\varepsilon\left(\frac{\partial C}{\partial t}\right)$.

However, the Clark model has successfully predicted a variety of systems and even those not following this assumption. As mentioned above, the dynamic adsorption is such a complicated process that it is almost impossible to give a complete description of each variable. Actually, even the "most theoretically rigorous" general rate models are still simplified from the real situations, such as the distribution of adsorbent pellets with different size in the column, the wall effect, the mass transfer caused by momentum transfer and heat transfer. Moreover, by mathematical fitting, each phenomenological coefficient could be adjusted to the optimal values, which would compensate for the inherent shortages of the model to some extent. Thus, it is still rational to suppose the Clark model has good performance under different conditions.

\subsection{Thomas model}

The Thomas model is another one frequently applied to estimate the adsorptive capacity of adsorbent and predict breakthrough curves, assuming the second-order reversible reaction kinetics and the Langmuir isotherm (Han et al., 2008; Ghasemi et al., 2011). Theoretically, it is suitable to estimate the adsorption process where external and internal diffusion resistances are extremely small (Aksu and Gönen, 2004). The Thomas model is given by

$$
\ln \left(\frac{C_{\mathrm{F}}}{C}-1\right)=\frac{k_{\mathrm{Th}} q_{\mathrm{F}} m}{Q}-k_{\mathrm{Th}} C_{\mathrm{F}} t
$$

where $k_{\mathrm{Th}}$ is the Thomas rate constant, $m$ is the mass of adsorbent in the column. With several couples of $m$ and $Q, k_{\mathrm{Th}}$ and $q_{\mathrm{F}}$ values derived through a plot of $\ln \left[\left(C_{\mathrm{F}} / C\right)-1\right]$ vs. $t$, further prediction and design is then available. Eq. (65) can also be expressed as

$$
\ln \left(\frac{C_{\mathrm{F}}}{C}-1\right)=k^{\prime}\left(t-t_{1}\right)
$$

where $k^{\prime}=k_{\mathrm{Th}} C_{\mathrm{F}}$ and $t_{1}=q_{\mathrm{F}} m /\left(Q C_{\mathrm{F}}\right)$. The general version of Eq. (66) is represented as (Pearl, 1977; Lin et al., 2002)

$$
\ln \left(\frac{C_{\mathrm{F}}}{C}-1\right)=b_{\mathrm{o}}+b_{1} t+b_{2} t^{2}+\cdots=\sum_{i} b_{i} t^{i}
$$

This equation is applied when $\ln \left[\left(C_{\mathrm{F}} / C\right)-1\right]$ vs. $t$ is not in linear form. By fitting the experimental data, the corresponding parameters $b_{i}$ can be calculated. Generally, it is adequately accurate to employ the former three terms. It is worth noting that $q_{\mathrm{F}}$ derived from the experiment is often conspicuously different from the value acquired by equilibrium calculation, and the bed adsorptive capacity is often determined from the dynamic adsorption (Brauch and Schlunder, 1975; McKay, 1984; McKay and Al-Duri, 1988; Ko et al., 2000).

\subsection{Bohart-Adams model and bed depth service time (BDST) model}

Bohart and Adams (1920) came up with the Bohart-Adams (B-A) model when they proceeded with their work of analyzing the typical chlorinecharcoal transmission curve. They hypothesized that the uptake rate of chlorine is proportional to the concentration of the chlorine existing in the bulk fluid and the residual adsorptive capacity of charcoal, from which the following two equations are obtained:

$$
\begin{aligned}
& \frac{\partial C}{\partial z}=-\frac{k_{\mathrm{B}} q_{\mathrm{r}} C}{u}, \\
& \frac{\partial q_{\mathrm{r}}}{\partial t}=-k_{\mathrm{B}} q_{\mathrm{r}} C,
\end{aligned}
$$

where $q_{\mathrm{r}}$ is the residual adsorptive capacity, and $k_{\mathrm{B}}$ is the kinetic constant of the Bohart-Adam model.

Additionally

$$
\left\{\begin{array}{l}
t=0 \rightarrow \frac{q_{\mathrm{r}}}{q_{\mathrm{m}}}=1, \\
z=0 \rightarrow \frac{C}{C_{\mathrm{F}}}=1 .
\end{array}\right.
$$

Finally, the basic form of the B-A model was obtained:

$$
\ln \left(\frac{C_{\mathrm{F}}}{C}-1\right)=\ln \left[\exp \left(k_{\mathrm{B}} q_{\mathrm{m}} \frac{H}{u}\right)-1\right]-k_{\mathrm{B}} C_{\mathrm{F}} t
$$

If $\exp \left(k_{\mathrm{B}} q_{\mathrm{m}} H / u\right)$ is much larger than 1, Eq. (70) can be reduced and rearranged as

$$
t=\frac{q_{\mathrm{m}}}{C_{\mathrm{F}} u} H-\frac{1}{k_{\mathrm{B}} C_{\mathrm{F}}} \ln \left(\frac{C_{\mathrm{F}}}{C}-1\right) .
$$


Eq. (71) is the so-called bed depth service time model proposed by Hutchins (1973). Note that the B-A model (or bed depth service time (BDST) model) involves several important variables of adsorption system such as $C_{\mathrm{F}}, u, q_{\mathrm{m}}$, and $H$, and approximate estimation of the effect of each parameter from both models is achievable. As two widely used models in practice, the B-A and BDST models succeeded in predicting several breakthrough curves and optimizing the parameters, although it is relatively rough (Ayoob et al., 2007; Bhakat et al., 2007; Maji et al., 2007; Han et al., 2008; Srivastava et al., 2008).

Both the B-A and BDST models have seven parameters, among which $C_{\mathrm{F}}$ and $u$ can be determined before the dynamic adsorption experiment. For the B-A model, the height of the fixed bed (i.e., the height of packing adsorbent), $H$, is a constant and can be directly measured. Subsequently, different $C$ and corresponding $t$ are obtained through the dynamic adsorption experiment, and then a plot of $\ln \left(C_{\mathrm{F}} / C-1\right)$ vs. $t$ should theoretically be a straight line. After linear regression, $q_{\mathrm{m}}$ and $k_{\mathrm{B}}$ can be calculated from the tangent slope and intercept, respectively. In terms of calculated $q_{\mathrm{m}}$ and $k_{\mathrm{B}}$, prediction of adsorption performance at different process variables is available based on Eq. (70). As for the application of the BDST model, similar to the B-A model, after determining the demand concentration of the effluent solution, $q_{\mathrm{m}}$ and $k_{\mathrm{B}}$ can be worked out by a straight line of $t$ vs. $H$. Then, the adsorption efficiency under various conditions can be predicted. Actually, $q_{\mathrm{m}}$ and $k_{\mathrm{B}}$ are not constant when the variable(s) is changed, which may lead to unsatisfactory prediction. Especially, at $50 \%$ breakthrough, $C / C_{\mathrm{F}}=0.5$ and $t=t_{1 / 2}$, and Eq. (71) changes to

$$
t_{1 / 2}=\frac{q_{\mathrm{m}} H}{C_{\mathrm{F}} u} .
$$

Hence, $q_{\mathrm{m}}$ can be calculated by plotting $t_{1 / 2}$ vs. $H$. By rearranging Eq. (71) into proper form or giving specific values to parameters, the BDST model is frequently employed as a powerful tool to find the optimal operation condition. More recently, Ko et al. (2000) came up with a method to optimize the BDST model, where the bed adsorptive capacity $q_{\mathrm{m}}$ is substituted by a modified correlation,

$$
q_{\mathrm{t}}=q_{\mathrm{m}}\left[\left(1-\exp \left(-a_{\mathrm{r}} \sqrt{t}\right)\right] .\right.
$$

Integrating into Eq. (68)

$$
t=m^{\prime}\left[\left(1-\exp \left(-a_{\mathrm{r}} \sqrt{t}\right)\right] z+b^{\prime},\right.
$$

where $q_{\mathrm{t}}$ is the adsorptive capacity of column at time $t$, $a_{\mathrm{r}}$ is the rate parameter, $m^{\prime}=q_{\mathrm{m}} /\left(C_{\mathrm{F}} u\right)$, and $b^{\prime}=$ $-\left[1 /\left(k_{\mathrm{B}} C_{\mathrm{F}}\right)\right] \ln \left[\left(C_{\mathrm{F}} / C\right)-1\right]$. This method provides a way to approximately evaluate the significance of both film diffusion and intrapellet diffusion. The intrapellet mass transfer rate is proportional to the square-root of the residence time when the sorption process is controlled by intrapellet diffusion (McKay, 1979),

$$
\frac{a_{1}}{a_{2}}=\sqrt{\frac{t_{1}^{\prime}}{t_{2}^{\prime}}}=\sqrt{\frac{u_{2}}{u_{1}}} .
$$

According to the correlation proposed by (Wilson and Geankoplis, 1966),

$$
S h=\frac{1.09}{\varepsilon} P e^{1 / 3} .
$$

Namely

$$
\frac{a_{1}}{a_{2}}=\left(\frac{u_{1}}{u_{2}}\right)^{2 / 3}=\left(\frac{u_{2}}{u_{1}}\right)^{-2 / 3} .
$$

Combining Eq. (75) and Eq. (77), one can obtain

$$
\frac{a_{1}}{a_{2}}=\left[\left(\frac{u_{2}}{u_{1}}\right)^{1 / 2}\right]^{p_{i}}\left[\left(\frac{u_{2}}{u_{1}}\right)^{-2 / 3}\right]^{p_{\mathrm{f}}}=\left(\frac{u_{2}}{u_{1}}\right)^{p^{\prime}},
$$

where $p_{\mathrm{i}}, p_{\mathrm{f}}$ reflect the fractions of the overall uptake rate influenced by intrapellet diffusion and film diffusion, respectively. Thus,

$$
\left\{\begin{array}{l}
0.5 p_{i}-\frac{2}{3} p_{\mathrm{f}}=p^{\prime} \\
p_{i}+p_{\mathrm{f}}=1
\end{array}\right.
$$

where $p^{\prime}$ is determined by fitting the experimental data so that $p_{\mathrm{i}}$ and $p_{\mathrm{f}}$ are acquired subsequently. 


\subsection{Yoon-Nelson model}

The Yoon-Nelson model is extremely concise in form, supposing that the decrease in the probability of each adsorbate to be adsorbed is proportional to the probability of its adsorption and breakthrough on the adsorbent (Yoon and James, 1984). It can be represented by

$$
\ln \frac{C}{C_{\mathrm{F}}-C}=K_{\mathrm{YN}} t-t_{1 / 2} K_{\mathrm{YN}}
$$

where $K_{\mathrm{YN}}$ is the Yoon-Nelson rate constant. By plotting $\ln \left[C /\left(C_{\mathrm{F}}-C\right)\right]$ vs. $t, K_{\mathrm{YN}}$ and theoretical $t_{1 / 2}$ are reckoned. The Yoon-Nelson model not only has a more simple form than other models, but also requires no detailed data concerning the characters of adsorbate and adsorbent, as well as the parameters of the fixed bed (Hamdaoui, 2006). Also, as limited by its rough form, the Yoon-Nelson model is less valuable or convenient to obtain process variables and to predict adsorption under variety conditions.

\subsection{Wang model}

Wang et al. (2003) developed a mass transfer model to describe the breakthrough curve of solutions containing Co or $\mathrm{Zn}$ ions in the fixed bed on the basis of the following assumptions

(1) The adsorption process remains isothermal;

(2) The mass transfer equation is written as

$$
-\frac{\mathrm{d} y}{\mathrm{~d} t}=k_{\mathrm{w}} x y,
$$

where $k_{\mathrm{w}}$ is the kinetic constant, $y$ is the fraction of the adsorbed metal ions, and $x$ is the fraction of metal ions passing through the fixed bed, with $x+y=1$.

(3) The breakthrough curve is symmetrical; and,

(4) There is negligible axial dispersion in the column.

Presuming $y=y_{\mathrm{w}}$ at $t=t_{\mathrm{w}}$ and integrating Eq. (80), one can obtain

$$
\ln \left[x\left(1-x_{\mathrm{a}}\right) / x_{\mathrm{a}}(1-x)\right]=k_{\mathrm{w}}\left(t_{\mathrm{a}}-t\right) .
$$

Let $w=0.5$, then $y_{\mathrm{w}}=y_{1 / 2}=x_{1 / 2}, t_{\mathrm{w}}=t_{1 / 2}$. Combining the above parameters with Eq. (81)

$$
t=t_{1 / 2}-\frac{1}{k_{\mathrm{w}}} \ln \left(\frac{1}{1-x}\right),
$$

where $x$ can be expressed as

$$
x=C / C_{\mathrm{F}} .
$$

Substituting Eq. (83) into Eq. (82), $t$ should be in direct proportion to $\ln \left[\left(C_{\mathrm{F}} / C\right)-1\right]$. A plot of $\ln \left[\left(C_{\mathrm{F}} / C\right)\right.$ $-1]$ vs. $t$ produces the slope and intercept value as $1 / k_{\mathrm{w}}$ and $t_{1 / 2}$, respectively. Then the entire breakthrough curve can be obtained based on Eq. (82). This model was successfully applied in some cases (Wang et al., 2003; Araneda et al., 2011). Meanwhile, similar to the Yoon-Nelson model, it cannot provide sufficient information of an adsorption system.

\subsection{Other models}

\subsubsection{Wolborska model}

Wolborska (1989a) and Wolborska and Pustelnik (1996) analyzed the adsorption of $p$-nitrophenol on activated carbon and found that the initial segment of the breakthrough curve is controlled by film diffusion with constant kinetic coefficient, and the concentration profile of the initial stage moves axially in the column at a constant velocity. Moreover, the width of concentration profile in the column and the final breakthrough curve were nearly constant. Based on above observations, they developed a model to describe the breakthrough at low concentration region, which was written as

$$
\ln \frac{C}{C_{\mathrm{F}}}=\frac{\beta_{\mathrm{L}} C_{\mathrm{F}} \varepsilon}{\rho q_{\mathrm{F}}} t-\frac{\beta_{\mathrm{L}} H}{u}
$$

where $\beta_{\mathrm{L}}$ is the film diffusivity, which can be determined from the initial linear section of the breakthrough curve in the semi-logarithmic system through experiment or correlations.

\subsubsection{Modified dose-response model}

This model was initially developed for pharmacology studies and recently used to describe adsorption of metals in some cases (Yan et al., 2001; Senthilkumar et al., 2006; Araneda et al., 2011). The modified dose-response model can be written as 


$$
\frac{C}{C_{\mathrm{F}}}=1-\frac{1}{1+\left(\frac{C_{\mathrm{F}} Q t}{q_{\mathrm{F}} m}\right)^{a^{\prime}}}
$$

After rearrangement, it could be written as

$$
\ln \frac{C}{C_{\mathrm{F}}-C}=a^{\prime} \ln \left(C_{\mathrm{F}} Q t\right)-a^{\prime} \ln \left(q_{\mathrm{F}} m\right),
$$

where $a^{\prime}$ is the model parameter. Similar to other models, $a^{\prime}$ and $q_{\mathrm{F}}$ could be determined by plotting $\ln \left[C /\left(C_{\mathrm{F}}-C\right)\right]$ vs. $\ln \left(C_{\mathrm{F}} Q t\right)$.

\section{Conclusions}

Fixed-bed or column adsorption is the most popular option in practical application of adsorption process, and due to the complexity of a column adsorption system and lack of solid theory, its mathematical modeling is obviously more difficult than batch adsorption. To choose or develop a suitable model, accuracy and convenience should be considered simultaneously. Currently, although some effort had been made to complete these models (especially the general rate models and LDF model), each model has its inherent shortages and requires further development. The general rate models (and "general rate type" models) and LDF model generally fit well with the experimental data for most cases, but they are relatively time-consuming. Other models including the wave propagation theory, Bohart-Adams model, Yoon-Nelson model, Thomas model, Wang model, Wolborska model, and modified dose-response model could be applicable without isotherm information, but they are derived from specific situations and limited in space. The Clark model is suitable to describe column adsorption obeying the Freundlich isotherm and do not show conspicuously better accuracy than the above models. The constant pattern model is relatively convenient to apply and gives satisfactory prediction, but it cannot be readily employed for adsorption predominated by intrapellet diffusion. In addition, experimental work is always required to determine the isotherm and dynamic model as well as the related parameters. Moreover, when the breakthrough curve deviates from the ideal S shape, prediction derived from any model usually cannot meet our demand.

\section{References}

Abu-Lail, L., Bergendahl, J.A., Thompson, R.W., 2012. Mathematical modeling of chloroform adsorption onto fixed-bed columns of highly siliceous granular zeolites. Environmental Progress \& Sustainable Energy, 31(4): 591-596. [doi:10.1002/ep.10593]

Abuzaid, N.S., Nakhla, G., 1997. Predictability of the homogeneous surface diffusion model for activated carbon adsorption kinetics; formulation of a new mathematical model. Journal of Environmental Science \& Health Part A, 32(7):1945-1961.

Aksu, Z., Gönen, F., 2004. Biosorption of phenol by immobilized activated sludge in a continuous packed bed: prediction of breakthrough curves. Process Biochemistry, 39(5):599-613. [doi:10.1016/S0032-9592(03)00132-8]

Araneda, C., Basualto, C., Sapag, J., Tapia, C., Cotoras, D., Valenzuela, F., 2011. Uptake of copper (II) ions from acidic aqueous solutions using a continuous column packed with microcapsules containing a $\beta$-hydroxyoximic compound. Chemical Engineering Research \& Design, 89(12):2761-2769. [doi:10.1016/j.cherd.2011.05.008]

Ayoob, S., Gupta, A., Bhakat, P.B., 2007. Analysis of breakthrough developments and modeling of fixed bed adsorption system for As (V) removal from water by modified calcined bauxite (MCB). Separation and Purification Technology, 52(3):430-438. [doi:10.1016/j. seppur.2006.05.021]

Azizian, S., 2004. Kinetic models of sorption: a theoretical analysis. Journal of Colloid and Interface Science, 276(1): 47-52. [doi:10.1016/j.jcis.2004.03.048]

Bhakat, P., Gupta, A., Ayoob, S., 2007. Feasibility analysis of As (III) removal in a continuous flow fixed bed system by modified calcined bauxite (MCB). Journal of Hazardous Materials, 139(2):286-292. [doi:10.1016/j.jhazmat.2006. 06.037]

Borba, C., Guirardello, R., Silva, E.A., Veit, M.T., Tavares, C.R.G., 2006. Removal of nickel (II) ions from aqueous solution by biosorption in a fixed bed column: Experimental and theoretical breakthrough curves. Biochemical Engineering Journal, 30(2):184-191. [doi:10.1016/j.bej. 2006.04.001]

Brauch, V., Schlunder, E., 1975. The scale-up of activated carbon columns for water purification, based on results from batch tests-II: Theoretical and experimental determination of breakthrough curves in activated carbon columns. Chemical Engineering Science, 30(5-6):539548. [doi:10.1016/0009-2509(75)80024-8]

Brunauer, S., Emmett, P.H., Teller, E., 1938. Adsorption of gases in multimolecular layers. Journal of the American Chemical Society, 60(2):309-319. [doi:10.1021/ ja01269a023]

Cheknane, B., Baudu, M., Bouras, O., Zermane, F., 2012. Modeling of basic green 4 dynamic sorption onto granular organo-inorgano pillared clays (GOICs) in column reactor. Chemical Engineering Journal, 209:7-12. [doi:10.1016/ j.cej.2012.07.118]

Chern, J.M., Huang, S.N., 1999. Study of nonlinear wave 
propagation theory. II. Interference phenomena of singlecomponent dye adsorption waves. Separation Science and Technology, 34(10):1993-2011. [doi:10.1081/SS100100751]

Chern, J.M., Chien, Y.W., 2002. Adsorption of nitrophenol onto activated carbon: isotherms and breakthrough curves. Water Research, 36(3):647-655. [doi:10.1016/S00431354(01)00258-5]

Chong, K., Volesky, B., 1995. Description of two-metal biosorption equilibria by Langmuir-type models. Biotechnology and Bioengineering, 47(4):451-460. [doi:10.1002/ bit.260470406]

Clark, R.M., 1987. Evaluating the cost and performance of field-scale granular activated carbon systems. Environmental Science \& Technology, 21(6):573-580. [doi:10. 1021/es00160a008]

Costa, C., Rodrigues, A., 1985. Design of cyclic fixed-bed adsorption processes. 1. Phenoal adsorption on polymeric adsorbents. AIChE Journal, 31(10):1645-1654. [doi:10. 1002/aic.690311008]

Crittenden, J.C., Weber, W.J., 1978. Predictive model for design of fixed-bed adsorbers: Parameter estimation and model development. Journal of the Environmental Engineering Division, 104(2):185-197.

Crittenden, J.C., Luft, C.P., Hand, D.W., 1985. Prediction of multicomponent adsorption equilibria in background mixtures of unknown composition. Water Research, 19(12):1537-1548. [doi:10.1016/0043-1354(85)90399-9]

Crittenden, J.C., Hutzler, N.J., Geyer, D.G., Oravitz, J.L., Friedman, G., 1986. Model development and parameter sensitivity. Water Resources Research, 22(3):271-284. [doi:10.1029/WR022i003p00271]

Cussler, E.L., 1976. Multicomponent Diffusion. Elsevier, Amsterdam.

Do, D., Mayfield, P., 1987. A new simplified model for adsorption in a single particle. AIChE Journal, 33(8): 1397-1400. [doi:10.1002/aic.690330819]

Du, X., Yuan, Q., Li, Y., 2008. Mathematical analysis of solanesol adsorption on macroporous resins using the general rate model. Chemical Engineering \& Technology, 31(9):1310-1318. [doi:10.1002/ceat.200800010]

Du, X., Yuan, Q., Zhao, J., Li, Y., 2007. Comparison of general rate model with a new model-artificial neural network model in describing chromatographic kinetics of solanesol adsorption in packed column by macroporous resins. Journal of Chromatography A, 1145(1-2):165-174. [doi:10.1016/j.chroma.2007.01.065]

Dubinin, M., Radushkevich, L., 1947. Equation of the characteristic curve of activated charcoal. Chemisches Zentralblatt, 1(1):875.

Finlayson, B., 2003. Nonlinear Analysis in Chemical Engineering. Ravenna Park Publishing, Inc.

Foo, K., Hameed, B., 2010. Insights into the modeling of adsorption isotherm systems. Chemical Engineering Journal, 156(1):2-10. [doi:10.1016/j.cej.2009.09.013]

Fournel, L., Mocho, P., Brown, R., le Cloirec, P., 2010. Modeling breakthrough curves of volatile organic compounds on activated carbon fibers. Adsorption, 16(3):147-153.
Ghasemi, M., Keshtkar, A.R., Dabbagh, R., Jaber Safdari, S., 2011. Biosorption of uranium (VI) from aqueous solutions by Ca-pretreated Cystoseira indicaalga: breakthrough curves studies and modeling. Journal of Hazardous Materials, 189(1-2):141-149 [doi:10.1016/j. jhazmat.2011.02.011]

Gholami, M., Talaie, M., 2010. Investigation of simplifying assumptions in mathematical modeling of natural gas dehydration using adsorption process and introduction of a new accurate LDF model. Industrial \& Engineering Chemistry Research, 49(2):838-846. [doi:10.1021/ ie901183q]

Giles, C., MacEwan, T., Nakhwa, S.N., 1960. Studies in adsorption. Part XI. A system of classification of solution adsorption isotherms, and its use in diagnosis of adsorption mechanisms and in measurement of specific surface areas of solids. Journal of the Chemical Society, (0):3973-3993. [doi:10.1039/jr9600003973]

Glueckauf, E., 1955. Theory of chromatography. Part 10.Formulæ for diffusion into spheres and their application to chromatography. Transactions of the Faraday Society, 51(0):1540-1551. [doi:10.1039/tf9555101540]

Gnielinski, V., 1978. Equations for calculation of heat and mass transfer in perfused ballasting of spherical particles at medium and high Peclet numbers. Verfahrenstechnik, 12(6):363-367.

Haghseresht, F., Lu, G., 1998. Adsorption characteristics of phenolic compounds onto coal-reject-derived adsorbents. Energy \& Fuels, 12(6):1100-1107. [doi:10.1021/ ef9801165]

Hamdaoui, O., 2006. Dynamic sorption of methylene blue by cedar sawdust and crushed brick in fixed bed columns. Journal of Hazardous Materials, 138(2):293-303. [doi:10.1016/j.jhazmat.2006.04.061]

Han, R., Ding, D., Xu, Y., Zou, W., Wang, Y., Li, Y., Zou, L., 2008. Use of rice husk for the adsorption of congo red from aqueous solution in column mode. Bioresource Technology, 99(8):2938-2946. [doi:10.1016/j.biortech. 2007.06.027]

Hand, D.W., Loper, S., Ari, M., Crittenden, J.C., 1985. Prediction of multicomponent adsorption equilibria using ideal adsorbed solution theory. Environmental Science \& Technology, 19(11):1037-1043. [doi:10.1021/es00141a002]

Heese, C., Worch, E., 1997. A new user-oriented method for prediction of intraparticle mass transfer coefficients for adsorber modelling. Vom Wasser, 89:373-391 (in German).

Helfferich, F., 1984. Conceptual view of column behavior in multicomponent adsorption or ion-exchange systems. AIChE Symposium Series, 80:1.

Helfferich, F.G., 1995. Ion Exchange, Dover Pubns.

Helfferich, F.G., Klein, G., 1970. Multicomponent Chromatography: Theory of Interference. M. Dekker, New York.

Helfferich, F.G., Carr, P.W., 1993. Non-linear waves in chromatography: I. Waves, shocks, and shapes. Journal of Chromatography A, 629(2):97-122. [doi:10.1016/00219673(93)87026-I]

Helfferich, F.G., Whitley, R.D., 1996. Non-linear waves in 
chromatography II. Wave interference and coherence in multicomponent systems. Journal of Chromatography A, 734(1):7-47. [doi:10.1016/0021-9673(95)01067-X]

Hutchins, R., 1973. New method simplifies design of activated carbon systems. Chemical Engineering, 80(19):133-138.

Jain, J.S., Snoeyink, V.L., 1973. Adsorption from bisolute systems on active carbon. Journal (Water Pollution Control Federation), 45(12):2463-2479.

Jia, Q., Lua, A.C., 2008. Concentration-dependent branched pore kinetic model for aqueous phase adsorption. Chemical Engineering Journal, 136(2-3):227-235. [doi:10.1016/j. cej.2007.04.005]

Kaczmarski, K., Antos, D., 1996. Fast finite difference method for solving multicomponent adsorption-chromatography models. Computers \& Chemical Engineering, 20(11): 1271-1276. [doi:10.1016/0098-1354(95)00247-2]

Kananpanah, S., Dizadji, N., Abolghasemi, H., Salamatinia, B., 2009. Developing a new model to predict mass transfer coefficient of salicylic acid adsorption onto IRA-93: Experimental and modeling. Korean Journal of Chemical Engineering, 26(5):1208-1212. [doi:10.1007/s11814-0090215-6]

Kataoka, T., Yoshida, H., Ueyama, K., 1972. Mass transfer in laminar region between liquid and packing material surface in the packed bed. Journal of Chemical Engineering of Japan, 5(2):132-136. [doi:10.1252/jcej. 5.132]

Ko, D.C.K., Porter, J.F., McKay, G., 2000. Optimised correlations for the fixed-bed adsorption of metal ions on bone char. Chemical Engineering Science, 55(23): 5819-5829. [doi:10.1016/S0009-2509(00)00416-4]

Ko, D.C.K., Porter, J.F., McKay, G., 2001. Film-pore diffusion model for the fixed-bed sorption of copper and cadmium ions onto bone char. Water Research, 35(16):3876-3886. [doi:10.1016/S0043-1354(01)00114-2]

Ko, D.C.K., Porter, J.F., McKay, G., 2002. A branched pore model analysis for the adsorption of acid dyes on activated carbon. Adsorption, 8(3):171-188. [doi:10.1023/ A:1021283731952]

Ko, D.C.K., Porter, J.F., McKay, G., 2003. Mass transport model for the fixed bed sorption of metal ions on bone char. Industrial \& Engineering Chemistry Research, 42(14):3458-3469. [doi:10.1021/ie020505t]

Ko, D.C.K., Porter, J.F., McKay, G., 2005. Application of the concentration-dependent surface diffusion model on the multicomponent fixed-bed adsorption systems. Chemical Engineering Science, 60(20):5472-5479. [doi:10.1016/j. ces.2005.04.048]

Langmuir, I., 1916. The constitution and fundmental properties of solids and liquids. part I. Solids. Journal of the American Chemical Society, 38(11):2221-2295. [doi:10. 1021/ja02268a002]

Lee, V.K.C., McKay, G., 2004. Comparison of solutions for the homogeneous surface diffusion model applied to adsorption systems. Chemical Engineering Journal, 98(3): 255-264. [doi:10.1016/j.cej.2003.08.028]

Lee, V.K.C., Porter, J.F., McKay, G., Mathews, A.P., 2005. Application of solid-phase concentration-dependent
HSDM to the acid dye adsorption system. AIChE Journal, 51(1):323-332. [doi:10.1002/aic.10290]

Lin, S.H., Wang, C.S., Chang, C.H., 2002. Removal of methyl tert-butyl ether from contaminated water by macroreticular resin. Industrial \& Engineering Chemistry Research, 41(16):4116-4121. [doi:10.1021/ie011020s]

Liu, B., Zeng, L., Ren, Q., 2010. Simulation of levulinic acid adsorption in packed beds using parallel pore/surface diffusion model. Chemical Engineering \& Technology, 33(7):1146-1152. [doi:10.1002/ceat.201000147]

Lo, I., Alok, P.A., 1996. Computer simulation of activated carbon adsorption for multi-component systems. Environment International, 22(2):239-252. [doi:10.1016/ 0160-4120(96)00009-8]

Maji, S.K., Pal, A., Pal, T., Adak, A., 2007. Modeling and fixed bed column adsorption of As (III) on laterite soil. Separation and Purification Technology, 56(3):284-290. [doi:10.1016/j.seppur.2007.02.011]

Malek, A., Farooq, S., 1996. Comparison of isotherm models for hydrocarbon adsorption on activated carbon. AIChE Journal, 42(11):3191-3201. [doi:10.1002/aic.690421120]

Mathews, A., Weber, W.J.J., 1977. Effects of external mass transfer and intraparticle diffusion on adsorption rates in slurry reactors. AIChE Symposium Series, 73:91-94.

McKay, G., 1979. Basic dye adsorption on activated carbon. Water, Air, \& Soil Pollution, 12(3):307-317. [doi:10. 1007/BF01047018]

McKay, G., 1984. Analytical solution using a pore diffusion model for a pseudoirreversible isotherm for the adsorption of basic dye on silica. AIChE Journal, 30(4): 692-697. [doi:10.1002/aic.690300434]

McKay, G., 2001. Solution to the homogeneous surface diffusion model for batch adsorption systems using orthogonal collocation. Chemical Engineering Journal, 81(1-3):213-221. [doi:10.1016/S1385-8947(00)00191-1]

McKay, G., Al-Duri, B., 1988. Prediction of bisolute adsorption isotherms using single-component data for dye adsorption onto carbon. Chemical Engineering Science, 43(5):1133-1142. [doi:10.1016/0009-2509(88)85073-5]

Medved, I., Cerny, R., 2011. Surface diffusion in porous media: A critical review. Microporous and Mesoporous Materials, 142(2-3):405-422.

Meng, M.J., Wang, Z.P., Ma, L., Zhang, M., Wang, J., Dai, X.H., Yan, Y.S., 2012. Selective adsorption of methylparaben by submicrosized molecularly imprinted polymer: batch and dynamic flow mode studies. Industrial \& Engineering Chemistry Research, 51(45):14915-14924. [doi:10.1021/ie301890b]

Murillo, R., García, T., Aylón, E., Callén, M.S., Navarro, M.V., López, J.M., Mastral, A.M., 2004. Adsorption of phenanthrene on activated carbons: Breakthrough curve modeling. Carbon, 42(10):2009-2017. [doi:10.1016/j. carbon.2004.04.001]

Myers, A., Prausnitz, J.M., 1965. Thermodynamics of mixedgas adsorption. AIChE Journal, 11(1):121-127. [doi:10. 1002/aic.690110125]

Nakao, S., Suzuki, M., 1983. Mass transfer coefficient in cyclic adsorption and desorption. Journal of Chemical 
Engineering of Japan, 16(2):114-119. [doi:10.1252/ jcej.16.114]

Neretnieks, I., 1976. Adsorption in finite bath and countercurrent flow with systems having a concentration dependant coefficient of diffusion. Chemical Engineering Science, 31(6):465-471. [doi:10.1016/0009-2509(76) 80031-0]

Nwabanne, J.T., Igbokwe, P.K., 2012. Kinetic modeling of heavy metals adsorption on fixed bed column. International Journal of Environmental Research, 6(4):945-952.

Ohashi, H., Sugawara, T., Kikuchi, K.I., Konno, H., 1981. Correlation of liquid-side mass transfer coefficient for single particles and fixed beds. Journal of Chemical Engineering of Japan, 14(6):433-438. [doi:10.1252/jcej. 14.433]

Pan, B.C., Meng, F.W., Chen, X.Q., Pan, B.J., Li, X.T., Zhang, W.M., Zhang, X., Chen, J.L., Zhang, Q.X., Sun, Y., 2005. Application of an effective method in predicting breakthrough curves of fixed-bed adsorption onto resin adsorbent. Journal of Hazardous Materials, 124(1-3): 74-80. [doi:10.1016/j.jhazmat.2005.03.052]

Pearl, R., 1977. The Biology of Population Growth. Ayer Co. Pub.

Peel, R.G., Benedek, A., Crowe, C.M., 1981. A branched pore kinetic model for activated carbon adsorption. AIChE Journal, 27(1):26-32. [doi:10.1002/aic.690270106]

Plazinski, W., Rudzinski, W., Plazinska, A., 2009. Theoretical models of sorption kinetics including a surface reaction mechanism: a review. Advances in Colloid and Interface Science, 152(1-2):2-13. [doi:10.1016/j.cis.2009.07.009]

Puértolas, B., López, M.R., Navarro, M.V., López, J.M., Murillo, R., García, T., Mastra, A.M., 2010. Modelling the breakthrough curves obtained from the adsorption of propene onto microporous inorganic solids. Adsorption Science \& Technology, 28(8):761-775. [doi:10.1260/ 0263-6174.28.8-9.761]

Quek, S., Al-Duri, B., 2007. Application of film-pore diffusion model for the adsorption of metal ions on coir in a fixed-bed column. Chemical Engineering and Processing Process Intensification, 46(5):477-485. [doi:10.1016/j. cep.2006.06.019]

Radke, C., Prausnitz, J., 1972. Thermodynamics of multisolute adsorption from dilute liquid solutions. AIChE Journal, 18(4):761-768. [doi:10.1002/aic.690180417]

Roberts, P.V., Cornel, P., Summers, R.S., 1985. External mass-transfer rate in fixed-bed adsorption. Journal of Environmental Engineering, 111(6):891-905. [doi:10. 1061/(ASCE)0733-9372(1985)111:6(891)]

Ruthven, D.M., 1984. Principles of Adsorption and Adsorption Processes. Wiley-Interscience.

Scott, D., Dullien, F., 1962. Diffusion of ideal gases in capillaries and porous solids. AIChE Journal, 8(1):113117. [doi:10.1002/aic.690080126]

Seidel-Morgenstern, A., 2004. Experimental determination of single solute and competitive adsorption isotherms. Journal of Chromatography A, 1037(1-2):255-272. [doi:10.1016/j.chroma.2003.11.108]

Senthilkumar, R., Vijayaraghavan, K., Thilakavathi, M., Iyer,
P.V.R., Velan, M., 2006. Seaweeds for the remediation of wastewaters contaminated with zinc (II) ions. Journal of Hazardous Materials, 136(3):791-799. [doi:10.1016/j. jhazmat.2006.01.014]

Silva, E.A., Vaz, L.G.L., Veit, M.T., Fagundes-Klen, M.R., Cossich, E.S., Tavares, C.R.G., Cardozo-Filho, L., Guirardello, R., 2010. Biosorption of chromium (III) and copper (II) ions onto marine Alga Sargassum sp. in a fixed-bed column. Adsorption Science \& Technology, 28(5):449-464. [doi:10.1260/0263-6174.28.5.449]

Sips, R., 1948. Combined form of Langmuir and Freundlich equations. The Journal of Chemical Physics, 16(5): 490-495. [doi:10.1063/1.1746922]

Sperlich, A., Werner, A., Genz, A., Amy, G., Worch, E., Jekel, M., 2005. Breakthrough behavior of granular ferric hydroxide (GFH) fixed-bed adsorption filters: modeling and experimental approaches. Water Research, 39(6): 1190-1198. [doi:10.1016/j.watres.2004.12.032]

Srivastava, V., Prasad, B., Mishra, I.M., Mall, I.D., Swamy, M.M., 2008. Prediction of breakthrough curves for sorptive removal of phenol by bagasse fly ash packed bed. Industrial \& Engineering Chemistry Research, 47(5): 1603-1613. [doi:10.1021/ie0708475]

Szukiewicz, M.K., 2000. New approximate model for diffusion and reaction in a porous catalyst. AIChE Journal, 46(3):661-665. [doi:10.1002/aic.690460326]

Szukiewicz, M.K., 2002. An approximate model for diffusion and reaction in a porous pellet. Chemical Engineering Science, 57(8):1451-1457. [doi:10.1016/S0009-2509(02) 00055-6]

Tan, A.Y., Prasher, B.D., Guin, J.A., 1975. Mass transfer in nonuniform packing. AIChE Journal, 21(2):396-397. [doi:10.1002/aic.690210226]

Temkin, M., Pyzhev, V., 1940. Kinetics of ammonia synthesis on promoted iron catalysts. Acta Physiochimica URSS, 12: 327-356.

Tien, C., 1994. Adsorption Calculations and Modeling. Butterworth-Heinemann Boston.

Toth, J., 1971. State equations of the solid-gas interface layers. Acta Chimica Academiae Scientiarum Hungaricae, 69(3): 311-328.

Traylor, S.J., Xu, X., Lenhoff, A.M., 2011. Shrinking core modeling of binary chromatographic breakthrough. Journal of Chromatography A, 1218(16):2222-2231. [doi:10.1016/j.chroma.2011.02.020]

Wakao, N., Funazkri, T., 1978. Effect of fluid dispersion coefficients on particle-to-fluid mass transfer coefficients in packed beds: Correlation of Sherwood numbers. Chemical Engineering Science, 33(10):1375-1384. [doi:10.1016/0009-2509(78)85120-3]

Wang, Y.H., Lin, S.H., Juang, R.S., 2003. Removal of heavy metal ions from aqueous solutions using various low-cost adsorbents. Journal of Hazardous Materials, 102(2-3): 291-302. [doi:10.1016/S0304-3894(03)00218-8]

Warchoł, J., Petrus, R., 2006. Modeling of heavy metal removal dynamics in clinoptilolite packed beds. Microporous and Mesoporous Materials, 93(1-3):29-39. [doi:10.1016/j.micromeso.2006.01.021] 
Weber, W., Smith, E., 1987. Simulation and design models for adsorption processes. Environmental Science and Technology, 21(11):1040-1050. [doi:10.1021/es00164a002]

Wilke, C., Chang, P., 1955. Correlation of diffusion coefficients in dilute solutions. AIChE Journal, 1(2):264-270. [doi:10.1002/aic.690010222]

Williamson, J., Bazaire, K., Geankoplis, C.J., 1963. Liquidphase mass transfer at low Reynolds numbers. Industrial \& Engineering Chemistry Fundamentals, 2(2):126-129. [doi:10.1021/i160006a007]

Wilson, E., Geankoplis, C., 1966. Liquid mass transfer at very low Reynolds numbers in packed beds. Industrial \& Engineering Chemistry Fundamentals, 5(1):9-14. [doi:10. 1021/i160017a002]

Wolborska, A., 1989a. Adsorption on activated carbon of $p$-nitrophenol from aqueous solution. Water Research, 23(1):85-91. [doi:10.1016/0043-1354(89)90066-3]

Wolborska, A., 1989b. Determination of mass transfer coefficient adsorption in a fixed bed. Inzynieria Chemiczna I Procesowa, 4:545-556.

Wolborska, A., Pustelnik, P., 1996. A simplified method for determination of the break-through time of an adsorbent layer. Water Research, 30(11):2643-2650. [doi:10.1016/ S0043-1354(96)00166-2]

Worch, E., 1993. Eine neue gleichung zur berechnung von diffusionskoeffizienten gelöster stoffe. Vom Wasser, 81: 289-297 (in German).

Worch, E., 2008. Fixed-bed adsorption in drinking water treatment: a critical review on models and parameter estimation. Journal of Water Supply Research and Technology-AQUA, 57(3):171-183. [doi:10.2166/aqua. 2008.100]

Xue, W.B., Yi, A.H., Zhang, Z.Q., Tang, C.L., Zhang, X.C., Gao, J.M., 2009. A new competitive adsorption isothermal model of heavy metals in soils. Pedosphere, 19(2): 251-257. [doi:10.1016/S1002-0160(09)60115-6]

Yan, G., Viraraghavan, T., Chen, M., 2001. A new model for heavy metal removal in a biosorption column. Adsorption Science \& Technology, 19(1):25-43. [doi:10.1260/ 0263617011493953]

Yang, X.Y., Al-Duri, B., 2001. Application of branched pore diffusion model in the adsorption of reactive dyes on activated carbon. Chemical Engineering Journal, 83(1): 15-23. [doi:10.1016/S1385-8947(00)00233-3]

Yao, C., Tien, C., 1993. Approximations of uptake rate of spherical adsorbent pellets and their application to batch adsorption calculations. Chemical Engineering Science, 48(1):187-198. [doi:10.1016/0009-2509(93)80295-2]

Yi, H.H., Deng, H., Tang, X.L., Yu, Q.F., Zhou, X., Liu, H.Y., 2012. Adsorption equilibrium and kinetics for $\mathrm{SO}_{2}, \mathrm{NO}$, $\mathrm{CO}_{2}$ on zeolites FAU and LTA. Journal of Hazardous Materials, 203-204:111-117. [doi:10.1016/j.jhazmat.2011. 11.091]

Yoon, Y.H., James, H.N., 1984. Application of gas adsorption kinetics I. A theoretical model for respirator cartridge service life. The American Industrial Hygiene Association
Journal, 45(8):509-516. [doi:10.1080/152986684914 00197]

Yun, J.X., Yao, S.J., Lin, D.Q., Lu, M.H., Zhao, W.T., 2004. Modeling axial distributions of adsorbent particle size and local voidage in expanded bed. Chemical Engineering Science, 59(2):449-457. [doi:10.1016/j.ces.2003.10.009]

Zhang, R., Ritter, J.A., 1997. New approximate model for nonlinear adsorption and diffusion in a single particle. Chemical Engineering Science, 52(18):3161-3172. [doi:10.1016/S0009-2509(97)00124-3]

Zhao, Y., Shen, Y.M., Bai, L., Ni, S.Q., 2012. Carbon dioxide adsorption on polyacrylamide-impregnated silica gel and breakthrough modeling. Applied Surface Science, 261: 708-716. [doi:10.1016/j.apsusc.2012.08.085]

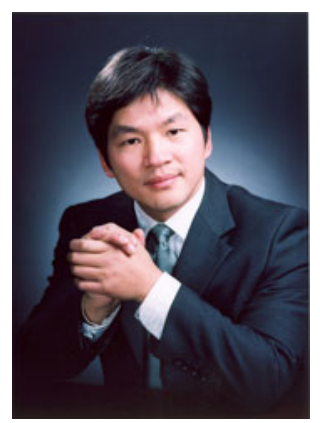

Introducing editorial board member:

Dr. Bing-cai PAN is the chair professor of Department of Environmental Engineering, Nanjing University, and the deputy director of State Key Laboratory of Pollution Control and Resource Reuse and National Engineering Research Center for Organic Pollution Control and Resource Reuse, China. He obtained his bachelor degree in Environmental Chemistry, and master and $\mathrm{PhD}$ degree in Environmental Engineering from Nanjing University. His current research activities include advanced treatment of water and wastewater, environmental functional materials, and environmental nanotechnology. Dr. PAN has authored more than 100 research papers in peer-reviewed journals, applied for 63 patents including 11 international patents. Due to his shining achievements in the related field, he was awarded the Asian Young Researcher Award in 2008 by the Conference of Asian University Presidents, the ProSPER.Net-Scopus Young Researcher Award in 2010, and the Jiangsu Young Scientist Award in 2012.

Currently, Dr. PAN serves as the chairman of the International Water Association (IWA) China Young Professionals, the responsible editor of Environmental Science and Pollution Research and Frontiers of Environmental Science and Engineering, and the editorial board member of several well-known journals, including Chemical Engineering Journal, Journal of Zhejiang University SCIENCE A (Applied Physics \& Engineering), and Ion Exchange \& Adsorption. 